\title{
New families of Sun-centered non-Keplerian orbits over cylinders and spheres
}

\author{
Jeannette Heiligers Colin R. McInnes
}

July 15, 2014

\begin{abstract}
This paper introduces new families of Sun-centered non-Keplerian orbits (NKOs) that are constrained to a three-dimensional, cylindrical or spherical surface. As such, they are an extension to the well-known families of displaced NKOs that are confined to a two-dimensional plane. The cylindrical and spherical orbits are found by investigating the geometrically constrained spacecraft dynamics. By imposing further constraints on the orbit's angular velocity and propulsive acceleration, the set of feasible orbits is defined. Additionally, the phase spaces of the orbits are explored and a numerical analysis is developed to find periodic orbits. The richness of the problem is further enhanced by considering both an inverse square acceleration law (mimicking solar electric propulsion) and a solar sail acceleration law to maintain the spacecraft on the three-dimensional surface. The wealth of orbits that these new families of NKOs generate allows for a range of novel space applications.
\end{abstract}

\section{Introduction}

By exploiting a continuous propulsive force, non-Keplerian orbits (NKOs) strongly perturb the two- or three body problem, thereby creating a wealth of new orbits for the spacecraft motion about the central body. A particular subset of non-Keplerian orbits in the two-body problem are the so-called displaced NKOs where the continuous acceleration is applied perpendicular to the orbit plane to displace the orbit away from the natural Keplerian orbit (McInnes, 1998, 2011; Mengali and Quarta, 2009). The dynamics of such two-body displaced NKOs have been investigated before by considering the spacecraft's equations of motion in a rotating frame of reference to make the problem autonomous (McInnes, 1997). Equilibrium solutions of these equations of motion then provide the sought for displaced NKOs and a transformation to an inertial frame will subsequently show that the spacecraft executes a circular orbit displaced away from the natural Keplerian orbit. The applications of such displaced NKOs are abundant and their potential has been shown in a range of studies, both Sun-centered and planet-centered. In the Sun-centered case, applications 
include solar physics and one year orbits synchronous with the Earth for space weather monitoring (McInnes and Simmons, 1992), while the planet-centered case includes applications such as displaced geostationary orbits to guarantee future geostationary slots for Earth observation and weather satellites (Baig and McInnes, 2010; Heiligers et al, 2011), hovering above Saturn's rings for highresolution imaging (Spilker, 2003), and NKOs displaced behind the Earth to observe the structure of the geomagnetic tail (Macdonald et al, 2006).

The objective of this paper is to extend these families of displaced NKOs, which are referred to in this paper as " $z$-static" (after their constant out-of-plane displacement), to families of NKOs that are confined to a cylindrical or spherical surface centered around the Sun, leaving the planet-centered case for future investigations. Feasible orbits will be found by constraining the dynamics of the spacecraft in a cylindrical or spherical coordinate system and imposing further constraints on the orbit angular velocity and acceleration magnitude. Furthermore, the system's phase space will be explored to identify new families of NKOs and Poincaré maps will be used to demonstrate the possible existence of periodic orbits. Finally, a numerical analysis is developed to find these periodic orbits. In order to generate the cylindrical and spherical NKOs, two types of propulsion will be considered: one where the acceleration is considered proportional to the Sun-spacecraft distance squared (mimicking solar electric propulsion (SEP)) and one that follows an ideal solar sail acceleration law. While SEP is a well-established propulsion technology with flight heritage on missions such as JAXA's Hayabusa (2003) (Kawaguchi et al, 2008), NASA's Dawn mission (2007) (Russell et al, 2004), and ESA's GOCE mission (2009) (Muzi and Allasio, 2004), solar sail technology is usually considered more far-term. Solar sailing exploits the radiation pressure generated by solar photons reflecting off a large, highly reflecting sail to produce a continuous thrust. Not constrained by propellant mass, solar sail missions have huge potential, which is reflected in ongoing solar sail activities. Recent advances include JAXA's IKAROS mission (2010) (Tsuda et al, 2011) and NASA's NanoSail-D2 mission (2010) (Johnson et al, 2011). Additionally, new solar sail initiatives are scheduled for the future, including NASA's Sunjammer mission ${ }^{1}$ and The Planetary Society's LightSail-1 mission (Biddy and Svitek, 2012). The required SEP and solar sail technology to enable the NKOs presented in this paper ranges from near-term to far-term, depending on the selected values for the design parameters. In some cases, a very high performance of the propulsion system is considered to illustrate the concept, but could eventually be feasible when advanced, high-performance technologies such as perforated sails become available (McInnes, 1999).

To introduce the novel cylindrical and spherical NKOs, the structure of the paper will be as follows. First, the cylindrical case will be presented: the equations of motion, control law and constraints required to maintain a spacecraft on a cylindrical surface will be obtained. Depending on a sign choice in the differential equation for the out-of-plane motion, two families of orbits can be

${ }^{1}$ L'Garde - Sunjammer, http://www.lgarde.com/programs/space-propulsion/sunjammer, Accessed 8 May 2013. 
distinguished, which will both be considered as well as the two different types of propulsive accelerations. For each case, the set of feasible orbits will be derived, example orbits will be shown and periodic orbits will be sought for. The paper will continue with a very similar analysis for the spherical case and ends with the conclusions.

\section{Cylindrically constrained NKOs}

Cylindrically constrained orbits can be found by considering the two-body equations of motion in a cylindrical coordinate system $(\rho, \theta, z)$ as shown in Figure 1. By assuming a central gravitational force field and an acceleration, $a$, in the $(\rho, z)$-plane only, the equations of motion can be written as:

$$
\begin{aligned}
\ddot{\rho}-\rho \dot{\theta}^{2} & =-\frac{\mu}{r^{3}} \rho+a \cos (\alpha+\gamma) \\
\rho^{2} \ddot{\theta}+2 \rho \dot{\rho} \dot{\theta} & =0 \\
\ddot{z} & =-\frac{\mu}{r^{3}} z+a \sin (\alpha+\gamma)
\end{aligned}
$$

with $\mu$ the gravitational parameter of the central body (here the Sun), $\alpha$ the acceleration angle with respect to the radial direction, also known as the cone angle, and $\gamma$ the elevation angle of the spacecraft.

To remain on a cylindrical surface, the following constraint on the projected radius $\rho$ is introduced:

$$
\rho=\text { constant } \rightarrow \ddot{\rho}(t)=\dot{\rho}(t)=0
$$

Furthermore, introducing the parameter $\bar{\omega}=\sqrt{\mu / \rho^{3}}$, which equals the angular velocity of a Keplerian orbit with radius $\rho$, the equations of motion reduce to:

$$
\begin{gathered}
\cos (\alpha+\gamma)=\frac{\rho \bar{\omega}^{2}}{a}\left(\left(1+\left(\frac{z}{\rho}\right)^{2}\right)^{-\frac{3}{2}}-\left(\frac{\omega}{\bar{\omega}}\right)^{2}\right) \\
\ddot{\theta}=0 \rightarrow \dot{\theta}=\text { constant }=\omega \\
\ddot{z}=-z \bar{\omega}^{2}\left(1+\left(\frac{z}{\rho}\right)^{2}\right)^{-\frac{3}{2}} \pm a \sqrt{1-\cos ^{2}(\alpha+\gamma)}
\end{gathered}
$$

Equation 3 provides the control law required for the spacecraft to stay on a cylindrical surface; equation 4 shows that the in-plane angular velocity is constant, which will be denoted by $\omega$; and Eq. 5 describes the spacecraft's motion in the out-of-plane direction. With initial conditions $z(0)=z_{0}, \dot{z}(0)=0$, this out-of-plane motion resembles a highly non-linear oscillator. Note that the plus-sign in Eq. 5 has been replaced by a \pm -sign as this generates two distinct families of cylindrical orbits. As will become clear in the next few sections, the minus-sign (which mirrors the acceleration in the $(x, y)$-plane) results in cylindrical orbits that oscillate around the $(x, y)$-plane, i.e. $-z_{0} \leq z(t) \leq z_{0}$, 


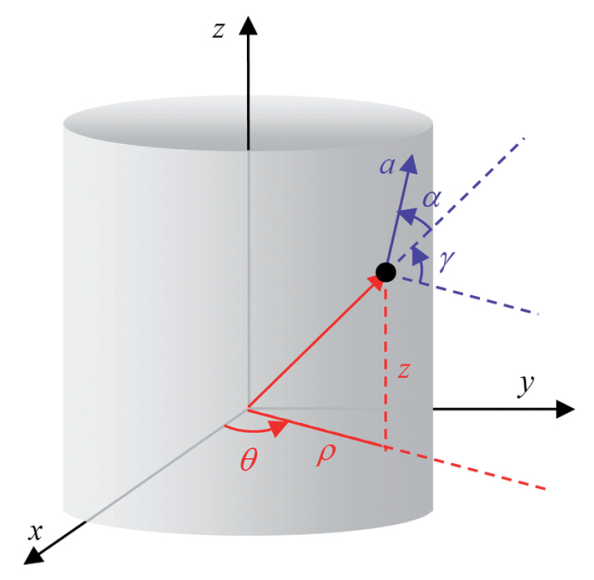

Figure 1: Definition of cylindrical reference frame.

while the plus-sign offers the possibility for the orbit to oscillate around a plane parallel to the $(x, y)$-plane. The family of orbits associated with the minus-sign will therefore hereafter be referred to as equatorial orbits, while the family of orbits associated with the plus-sign will be referred to as displaced orbits. They will first be considered for an inverse square acceleration law, followed by a very similar analysis for an ideal solar sail acceleration law.

\subsection{Inverse square acceleration law}

The inverse-square acceleration law is adopted to mimic the acceleration produced by an SEP system. For the Sun-centered case, a rule of thumb is to assume that the SEP acceleration scales with the distance to the Sun, $r$, squared:

$$
a=\beta_{S E P} \frac{\mu}{r^{2}}=\beta_{S E P} \bar{\omega}^{2} \rho\left(1+\left(\frac{z}{\rho}\right)^{2}\right)^{-1}
$$

with $\beta_{S E P}$ a scaling factor. Note that, throughout the paper, the influence of a changing spacecraft mass (due to propellant consumption) on the SEP acceleration is neglected.

Using the acceleration in Eq. 6, feasible orbits are found only if $-1 \leq$ $\cos (\alpha+\gamma) \leq 1$. Then, from Eq. 3, the following constraints on the in-plane angular velocity, $\omega$, and the scaling factor, $\beta_{S E P}$, can be derived:

$$
\omega \leq \omega_{\max }=\bar{\omega} \sqrt{\beta_{S E P}\left(1+\left(\frac{z}{\rho}\right)^{2}\right)^{-1}+\left(1+\left(\frac{z}{\rho}\right)^{2}\right)^{-\frac{3}{2}}}
$$




$$
\beta_{S E P} \geq \beta_{S E P, \text { min }}=\left(1+\left(\frac{z}{\rho}\right)^{2}\right)\left(\left(1+\left(\frac{z}{\rho}\right)^{2}\right)^{-\frac{3}{2}}-\left(\frac{\omega}{\bar{\omega}}\right)^{2}\right)
$$

Since $\rho, \omega, \bar{\omega}$ and $\beta_{S E P}$ are constant, both constraints vary only with the out-of-plane displacement, $z$. Since $z$ changes along the orbit, the values for $\omega$ and $\beta_{S E P}$ have to be chosen carefully to make sure that the constraints are satisfied throughout an entire oscillating motion in $z$. Since the minimum value for the right-hand side of Eq. 7 occurs at the maximum value for $z$, i.e. $z_{\max }$, the constraint on $\omega$ should be evaluated at $z_{\max }$. A similar reasoning can be adopted for the constraint on the scaling factor, $\beta_{S E P}$, in Eq. 8: the maximum value for the right-hand side in Eq. 8 occurs at the minimum absolute value for $z$, i.e. $|z|_{\text {min }}$. The constraint on $\beta_{S E P}$ should therefore be evaluated at $|z|_{\text {min }}$. The actual values for $|z|_{\min }$ and $z_{\max }$ depend on the family of orbits considered (i.e. equatorial or displaced) as will become clear in the following.

\subsubsection{Family of equatorial orbits}

As indicated above, by considering the minus sign in Eq. 5, orbits originate that oscillate around the $(x, y)$-plane such that $-z_{0} \leq z(t) \leq z_{0}$. Then, it is immediately clear that $|z|_{\min }=0$ and $z_{\max }=z_{0}$. The constraints in Eqs. 7 and 8 therefore reduce to:

$$
\begin{gathered}
\omega \leq \omega_{\max }=\bar{\omega} \sqrt{\beta_{S E P}\left(1+\left(\frac{z_{0}}{\rho}\right)^{2}\right)^{-1}+\left(1+\left(\frac{z_{0}}{\rho}\right)^{2}\right)^{-\frac{3}{2}}} \\
\beta_{S E P} \geq \beta_{S E P, \text { min }}=1-\left(\frac{\omega}{\bar{\omega}}\right)^{2}
\end{gathered}
$$

These constraints can be evaluated analytically, resulting in the region of feasible equatorial, cylindrical orbits with an inverse square acceleration law as shown in Figure 2 for $\rho=0.9$ and $z_{0}=0.5$, an example case that will be considered throughout this paper. Note that the results in the figure and any subsequent figures are made dimensionless by choosing the Sun's gravitational parameter $\mu$ to be unity and the unit of distance to be the astronomical unit. The non-dimensional unit of time then becomes $\frac{1}{2 \pi}$ years and an orbit with angular velocity $\omega=1$ is synchronous with the Earth.

A first impression of the type of equatorial, cylindrically constrained NKOs that are feasible is provided in Figure 3 for two particular values of the scaling factor, $\beta_{S E P}=0.32$ (plots a-b) and $\beta_{S E P}=0.44$ (plots c-d). The figures clearly show that these types of orbits oscillate around the $(x, y)$-plane and move between $z=z_{0}$ and $z=-z_{0}$. The figure also includes the Poincaré maps of the orbits, showing the system's phase space after each full revolution (with a maximum of 20 revolutions). These maps indicate that the orbits are quasiperiodic, especially the orbit with $\beta_{S E P}=0.44$. To find true periodic orbits, a numerical analysis is developed as described below. 


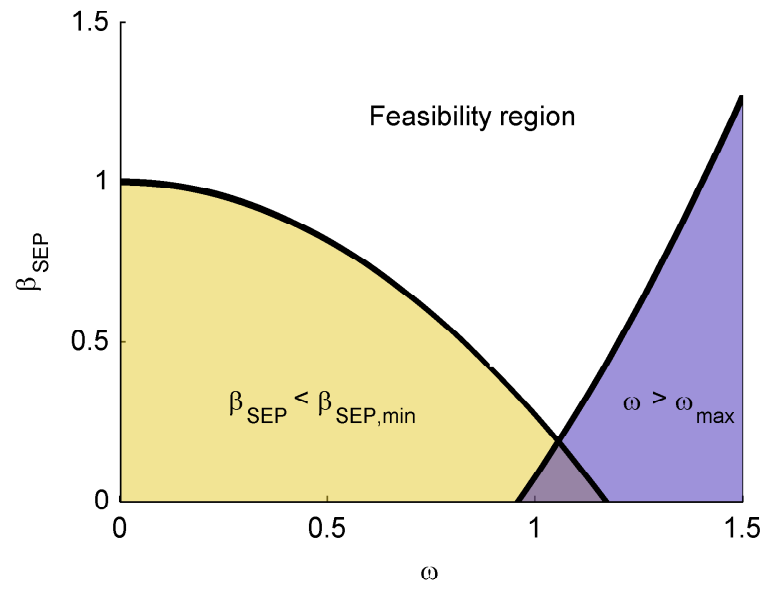

Figure 2: Feasibility region for equatorial, cylindrical NKOs with inverse-square acceleration law and for $\rho=0.9$ and $z_{0}=0.5$.

In order to obtain true periodic orbits, a numerical scheme is developed in which the initial conditions are integrated forward until the orbit crosses the $(x, y)$-plane. The angular distance at this crossing is denoted by $\theta=\theta_{z=0}$. Due to symmetry, a full period of the orbit extends over an angle, $\theta_{P}$, which equals $\theta_{P}=4 \theta_{z=0}$. This period can also be expressed as a fraction of a full revolution, i.e. $4 \theta_{z=0} / 2 \pi$, which equals the ratio of the out-of-plane angular velocity, $\omega_{z}$, and the in-plane angular velocity, $\omega: \omega_{z} / \omega=4 \theta_{z=0} / 2 \pi$. If this fraction equals an irreducible fraction (e.g. $\frac{1}{2}, \frac{2}{3}, \frac{4}{5}$, etc.) the orbit is periodic, where the period is given by the nominator of the irreducible fraction. An example is given in Figure 4, which provides the ratio of out-of-plane and in-plane angular velocities for $\rho=0.9$ and $z_{0}=0.5$ and for a range of in-plane angular velocities and scaling factors. The black line in the figure represents one particular ratio, $\omega_{z} / \omega=\frac{1}{2}$. Each intersection of the curved lines and this black line is a true periodic orbit with a period of one revolution. An example of such an orbit is given in Figure $4 \mathrm{~b}$, which corresponds to the black dot in Figure 4a. Through a fine grid search over values for $\omega$ and $\beta_{S E P}$ and by using an interpolation scheme to accurately determine the intersections between the $\omega_{z} / \omega$-curves and the lines of constant irreducible fractions, the periodic orbits as shown in Figure 5 are obtained. A maximum period of 10 revolutions is considered, implying that all irreducible fractions with a nominator of 1 to 10 are accounted for. The figure shows that lines of equal periods exist in the feasibility region of the equatorial, cylindrical orbits. Some examples of these true periodic orbits are shown in Figure 6, which correspond to the black dots in Figure 5. 


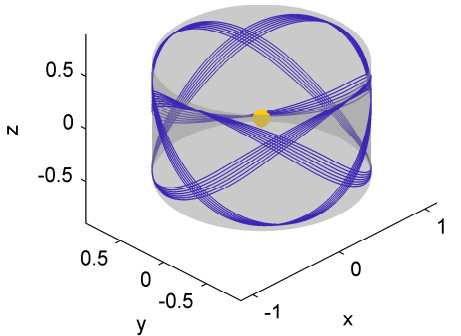

(a)

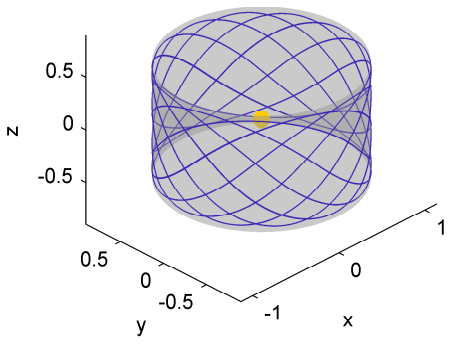

(c)

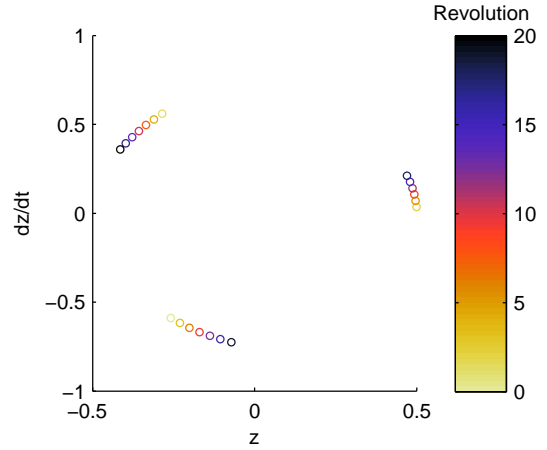

(b)

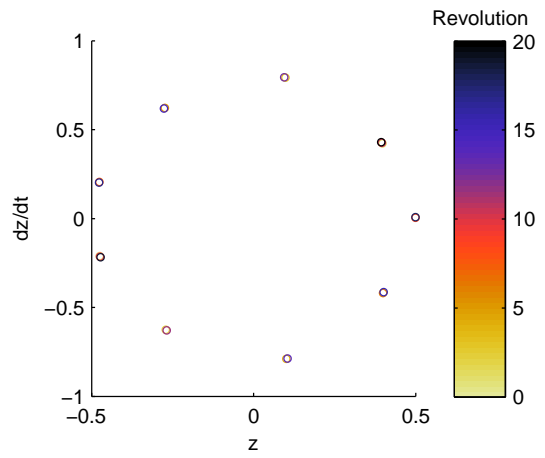

(d)

Figure 3: Orbital plots and Poincaré maps for equatorial, cylindrical NKOs with inverse-square acceleration law for $\rho=0.9, z_{0}=0.5$ and $\omega=1$. a-b) $\beta_{S E P}=0.32$. c-d) $\beta_{S E P}=0.44$. 


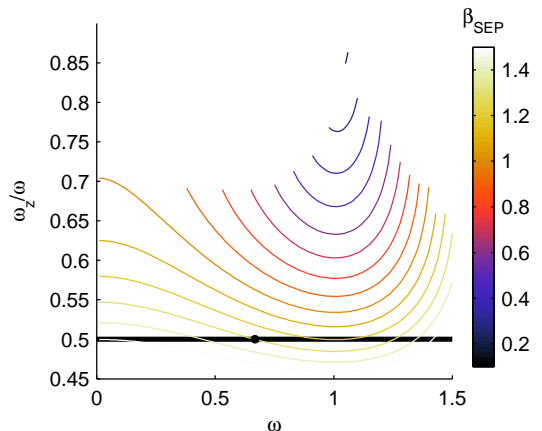

(a)

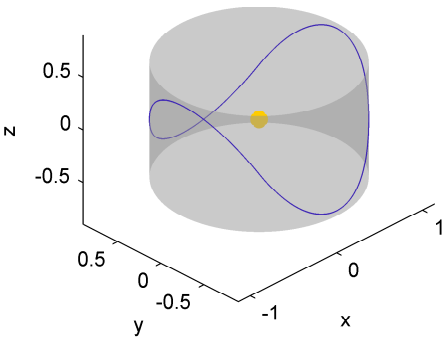

(b)

Figure 4: Equatorial, cylindrical NKOs with inverse-square acceleration law for $\rho=0.9$ and $z_{0}=0.5$. a) Ratio of out-of-plane and in-plane angular velocities. b) Example periodic orbit (see black dot in plot a)) with $\omega=0.6675$ and $\beta_{S E P}=$ 1.3 .

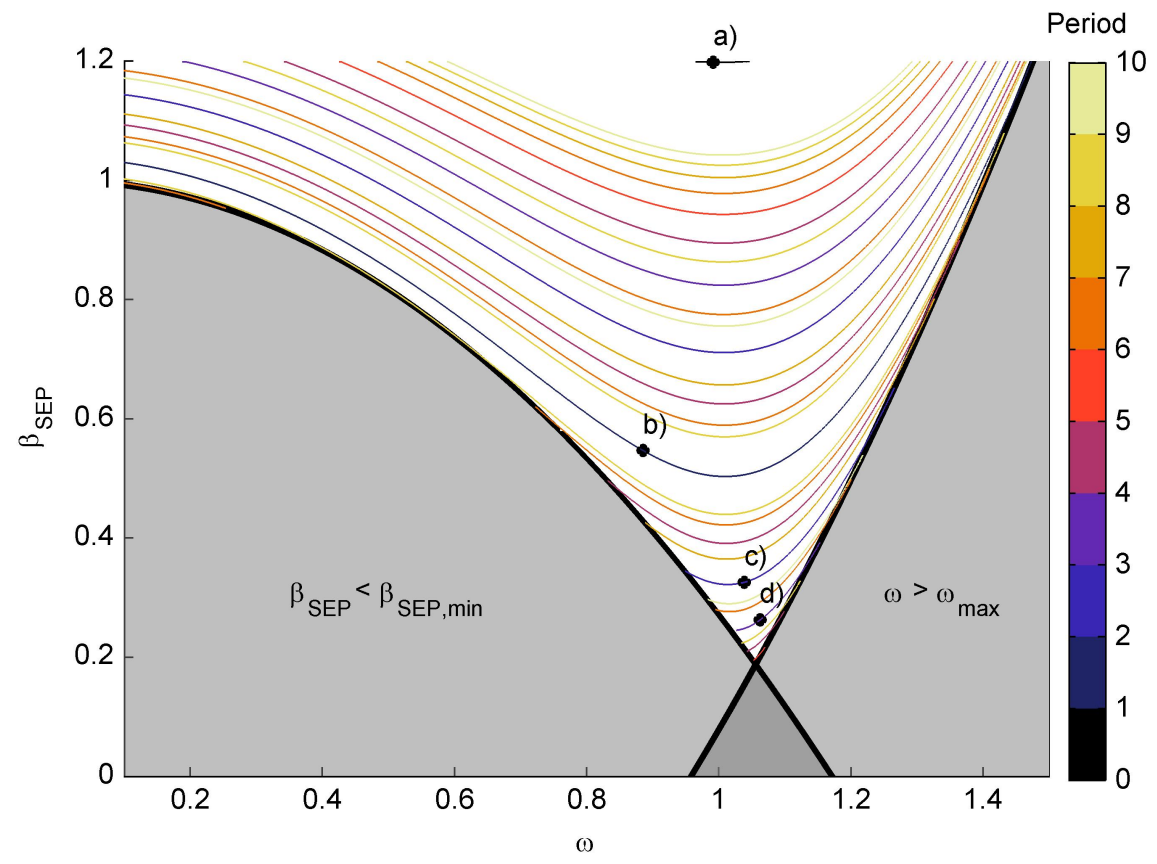

Figure 5: Periodic equatorial, cylindrical NKOs with inverse-square acceleration law for $\rho=0.9$ and $z_{0}=0.5$. The black dots correspond to the orbits in Figure 6. 

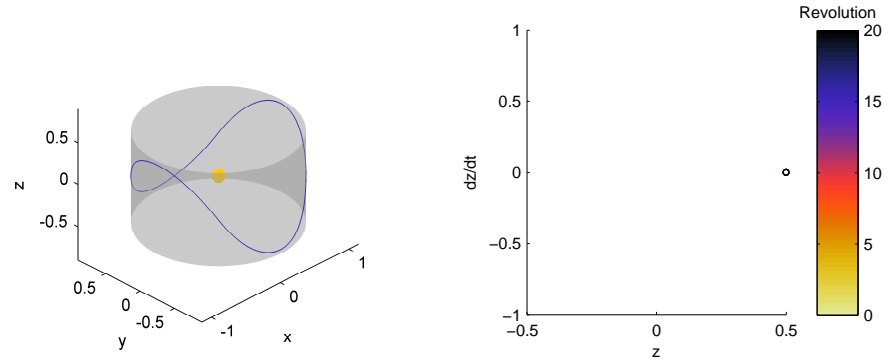

(a)
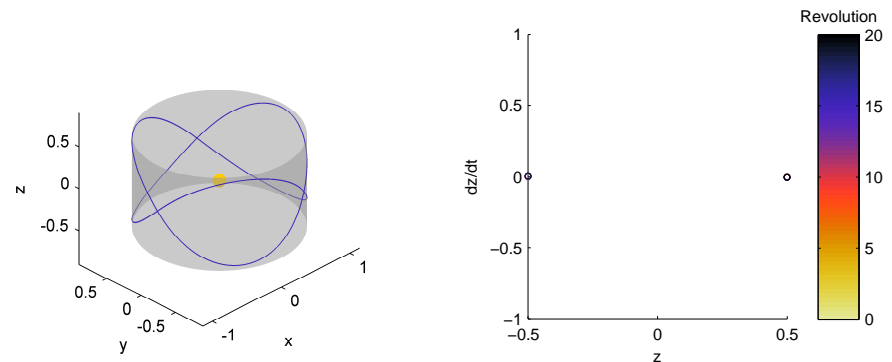

(b)
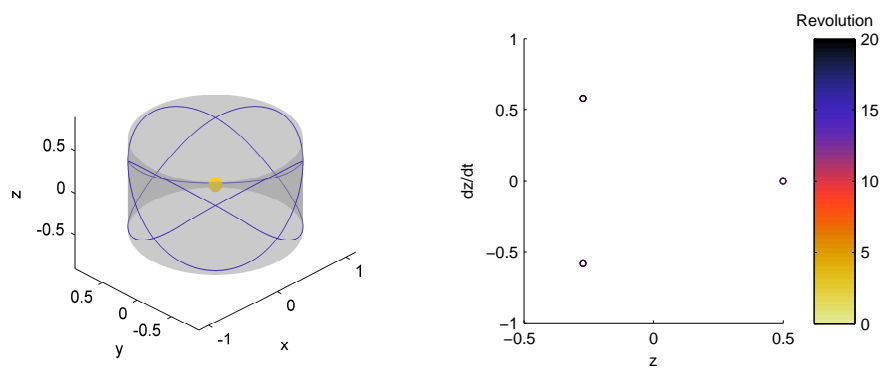

(c)
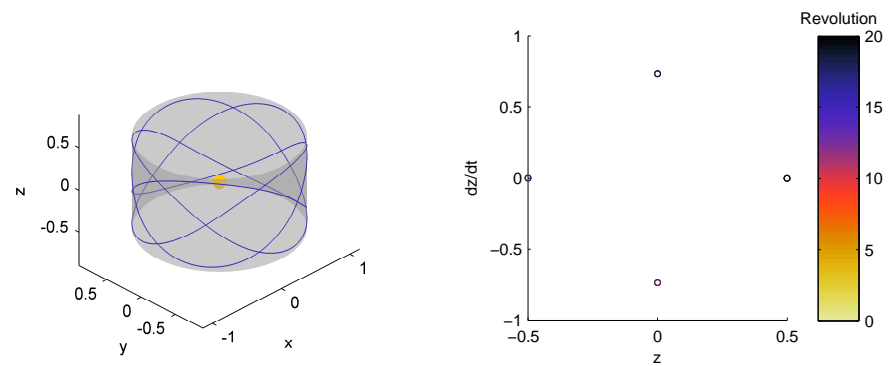

(d)

Figure 6: Periodic orbits (see black dots in Figure 5) and Poincaré maps for equatorial, cylindrical NKOs with inverse-square acceleration law for $\rho=0.9$ and $z_{0}=0.5$. a) $\omega=0.991, \beta_{S E P}=1.198$. b) $\omega=0.885, \beta_{S E P}=0.547$. c) $\omega=1.038, \beta_{S E P}=0.326$. d) $\omega=1.062, \beta_{S E P}=0.263$. 


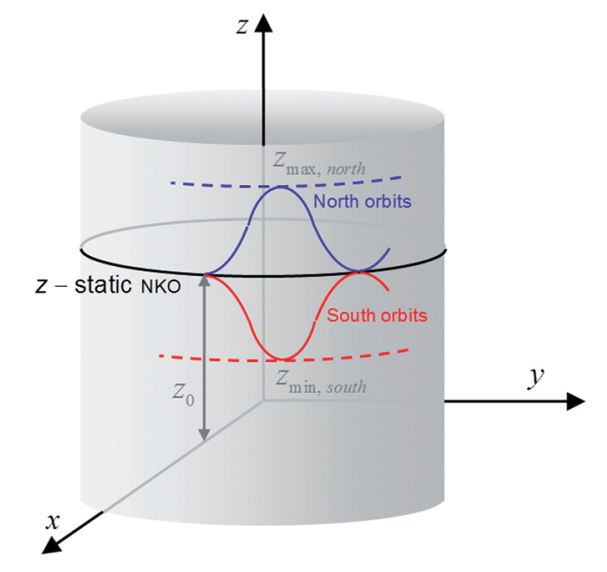

Figure 7: Definition of north and south displaced, cylindrical orbits.

\subsubsection{Family of displaced orbits}

When considering the plus-sign in Eq. 5, an additional family of cylindrical NKOs orbits can be found: the family of so-called displaced, cylindrical orbits. This family will include the well-known $z$-static displaced NKOs that were discussed in the introduction of this paper, since these $z$-static orbits can be considered to lie on a cylindrical surface. Furthermore, since these $z$-static NKOs maintain a constant out-of-plane displacement, $z(t)=z_{0}$ (i.e. $\ddot{z}(t)=\dot{z}(t)=0$ ), the required scaling factor, $\beta_{S E P, z}$, can be found by setting $\ddot{z}(t)=0$ in Eq. 5 :

$\beta_{S E P, z-\text { static }}=\sqrt{\left(1+\left(\frac{z_{0}}{\rho}\right)^{2}\right)^{-1}\left(\left(\frac{z_{0}}{\rho}\right)^{2}+\left(\left(\frac{\omega}{\bar{\omega}}\right)^{2}\left(1+\left(\frac{z_{0}}{\rho}\right)^{2}\right)^{\frac{3}{2}}-1\right)^{2}\right)}$

By substituting Eq. 11 into the constraints defined in Eqs. 7 and 8, it can be shown that these $z$-static NKOs are feasible for all $\omega$.

By increasing or decreasing $\beta_{S E P}$ with respect to $\beta_{S E P, z-s t a t i c}$, while still satisfying the control law in Eq. 3, the family of displaced, cylindrical NKOs can be found. As will become clear later, by increasing $\beta_{S E P}$ with respect to $\beta_{S E P, z-s t a t i c}$, orbits that lie entirely above the $z$-static NKO from which they bifurcate are created. This sub-family will be referred to as 'north orbits', see Figure 7 for an illustration. Contrary, by decreasing $\beta_{S E P}$ with respect to $\beta_{S E P, z-s t a t i c}$, a sub-family of orbits that lie entirely below the $z$-static NKO are created, which will be referred to as 'south orbits', see again Figure 7. This distinction has implications on the evaluation of the constraints in Eqs. 7 and 8 , as will be explained hereafter.

As stated before, to evaluate the maximum allowable value for $\omega$, the value for $z_{\max }$ is required, which is known for south orbits $\left(z_{\max }=z_{0}\right)$ but not for 
north orbits, since it is unknown a priori how much the north orbit will deviation from its initial conditions. For north orbits, the constraint on $\omega$ can therefore only be determined numerically through an integration of the orbit and by determining whether or not the maximum allowable in-plane angular velocity is exceeded. Instead, for south orbits (indicated by the subscript 'south'), the constraint can be evaluated analytically as:

$$
\omega_{\text {south }} \leq \omega_{\max }=\bar{\omega} \sqrt{\beta_{S E P}\left(1+\left(\frac{z_{0}}{\rho}\right)^{2}\right)^{-1}+\left(1+\left(\frac{z_{0}}{\rho}\right)^{2}\right)^{-\frac{3}{2}}}
$$

Contrary, to evaluate the minimum required value for $\beta_{S E P}$, the value of $|z|_{\min }$ is required, which is known for north orbits $\left(|z|_{\min }=z_{0}\right)$, but can again only be obtained through an integration of the orbit for south orbits. The analytical constraint on $\beta_{S E P}$ for north orbits (indicated by the subscript 'north') is:

$$
\beta_{S E P, \text { north }} \geq \beta_{S E P, \text { min }}=\left(1+\left(\frac{z_{0}}{\rho}\right)^{2}\right)\left(\left(1+\left(\frac{z_{0}}{\rho}\right)^{2}\right)^{-\frac{3}{2}}-\left(\frac{\omega}{\bar{\omega}}\right)^{2}\right)
$$

The result of a combined analytical and numerical computation of the feasibility region for displaced, cylindrical NKOs with an inverse square acceleration law and for the case $\rho=0.9$ and $z_{0}=0.5$ is given in Figure 8, where the thick red line dividing the feasibility region represents the value for $\beta_{S E P, z-s t a t i c}$. All feasible orbits above this line (i.e. $\beta_{S E P}>\beta_{S E P, z-\text { static }}$ ) are north orbits, those below the line are south orbits (i.e. $\beta_{S E P}<\beta_{S E P, z-s t a t i c}$ ). Furthermore, the figure includes the constraints in Eqs. 12 and 13 through the use of a dotted and dashed white line, respectively. Note that the dashed line, which gives the minimum value for $\beta_{S E P}$ for north orbits, lies below the thick red line for $\beta_{S E P, z-s t a t i c}$, again indicating that $z$-static NKOs are feasible for all $\omega$.

The feasibility region in Figure 8 can also be represented through the system's phase space, which gives an insight in the actual shape of the cylindrical orbits, see Figure 9. The phase space gives the out-of-plane motion, $z$, and outof plane velocity, $\dot{z}$, for each combination of $\omega$ (on $z$-axis) and $\beta_{S E P}$ (through the use of color). However, rather than using the absolute value for $\beta_{S E P}$, Figure 9 expresses the scaling factor as a percentage deviation from $\beta_{S E P, z-s t a t i c}$, $\Delta \beta_{S E P}$. Therefore, for $\Delta \beta_{S E P}=0, \beta_{S E P}=\beta_{S E P, z \text {-static }}$ (i.e. the $z$-static NKO case), which is represented by black dots on a grey transparent surface. Thus, the phase spaces to the right of this surface are north orbits, for which $\Delta \beta_{S E P}>0$, while phase spaces to the left of this surface are south orbits for which $\Delta \beta_{S E P}<0$. Some examples of both north and south orbits are provided in Figure 10, where the black lines indicate the $z$-static NKOs from which the cylindrical orbits bifurcate.

From the phase spaces in Figure 9 and the example orbits in Figure 10 it becomes clear that north and south orbits move in a confined band above or 


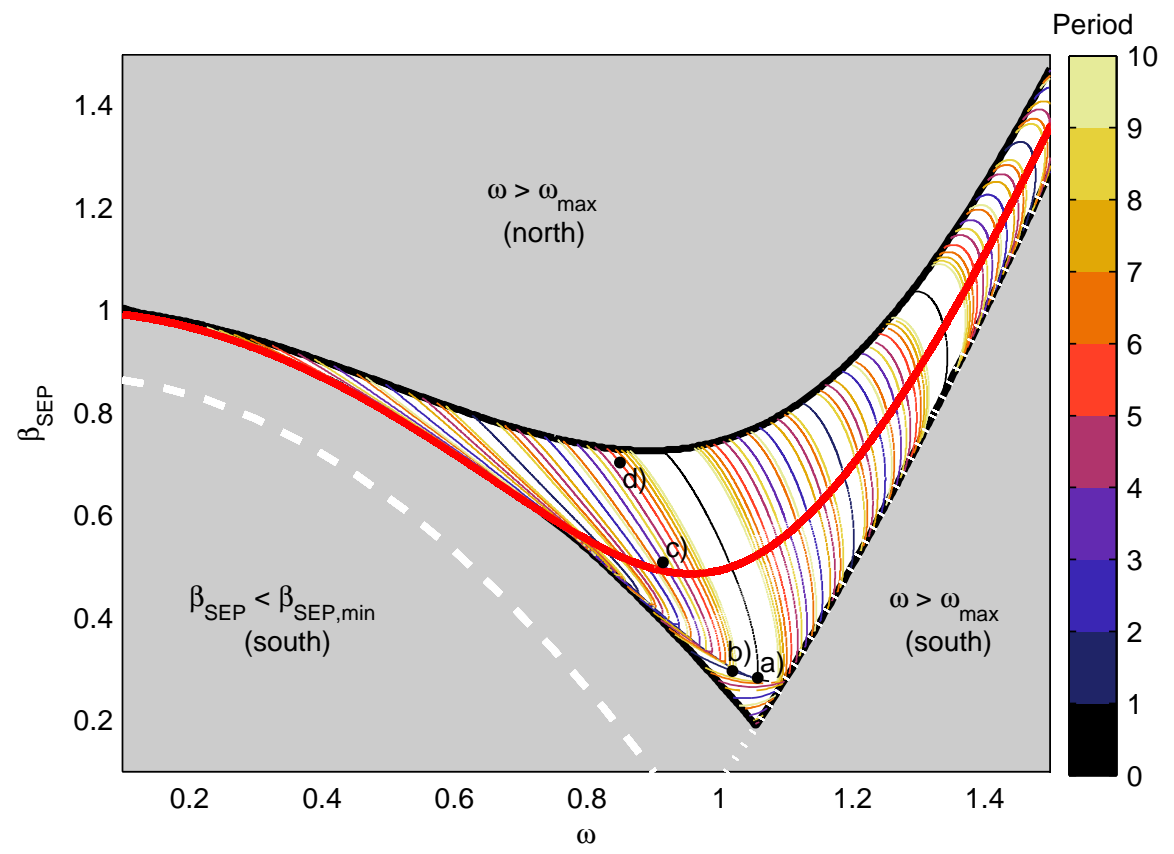

Figure 8: Feasibility region including periodic orbits for displaced, cylindrical NKOs with inverse-square acceleration law for $\rho=0.9$ and $z_{0}=0.5$. The thick red line dividing the feasibility region equals Eq. 11, the dotted white line equals Eq. 12, and the dashed white line equals Eq. 13. The black dots correspond to the orbits in Figure 11. 


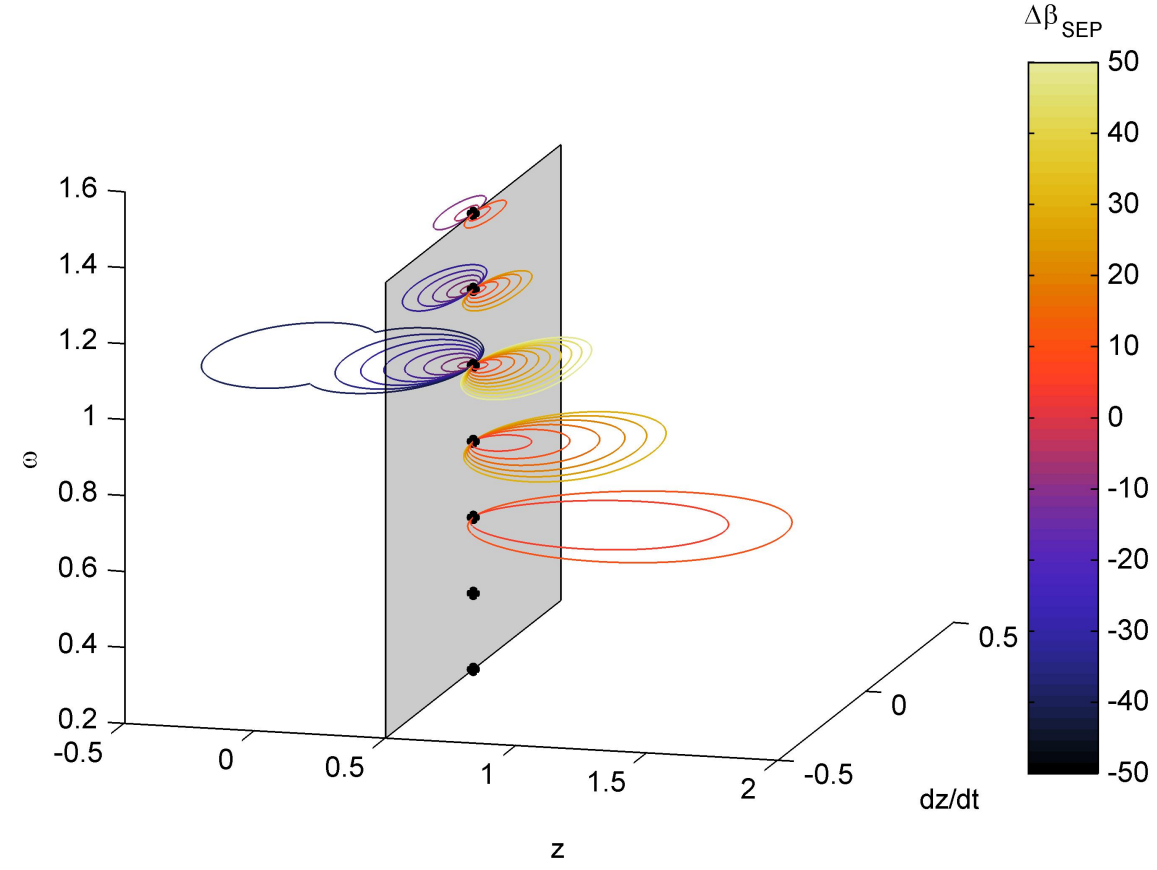

Figure 9: Phase spaces for displaced, cylindrical NKOs with inverse-square acceleration law for $\rho=0.9$ and $z_{0}=0.5$. Colors indicate percentage deviation

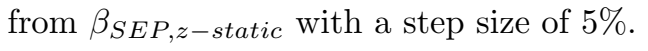



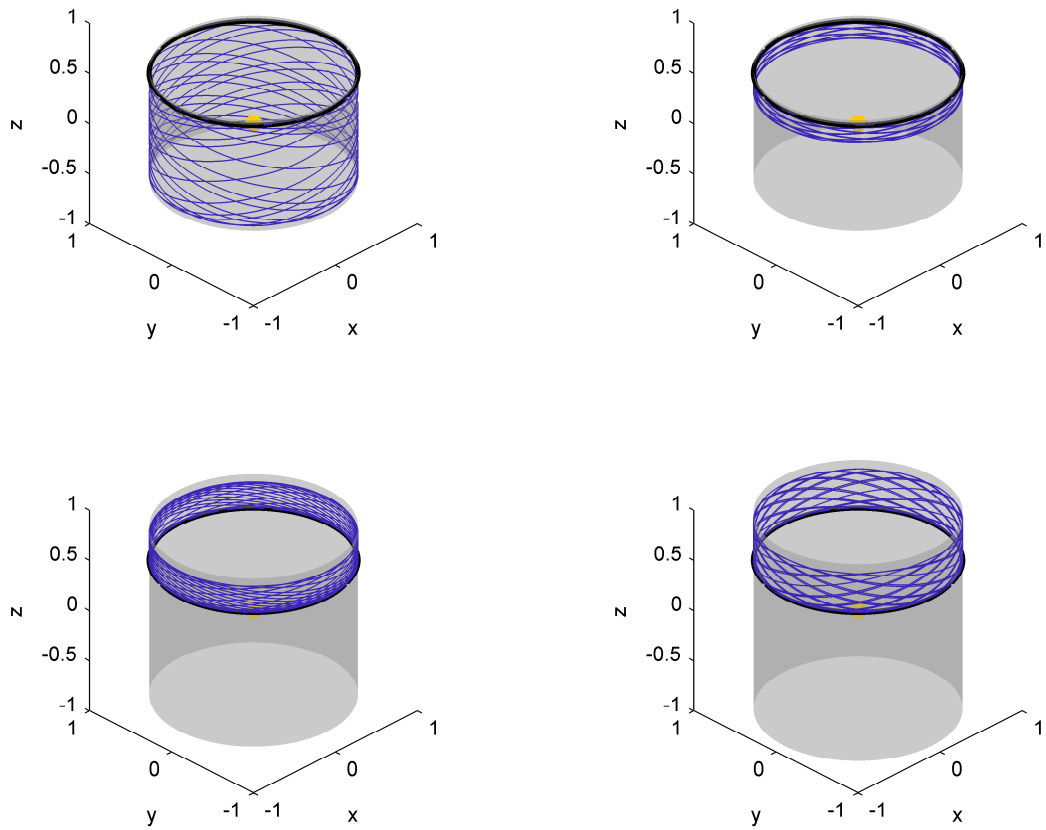

Figure 10: Examples of displaced, cylindrical NKOs with inverse-square acceleration law for $\rho=0.9, z_{0}=0.5$ and $\omega=1.0$ and for different values for $\Delta \beta_{S E P}$. Starting at top left plot and in clockwise direction: $\Delta \beta_{S E P}=-40 \%,-15 \%, 50 \%$ and $30 \%$. 
below the $z$-static NKO, respectively. Furthermore, for some cases, the value for $\beta_{S E P}$ can be decreased to such extent that the phase space crosses the $(x, y)$-plane after which it immediately transforms into the phase space of an equatorial orbit, i.e. $-z_{0} \leq z \leq z_{0}$. This is, for example, the case for $\omega=1.0$ and $\Delta \beta_{S E P}=-40 \%$ (see also the top left plot in Figure 10). The phase spaces finally show that, the larger $\Delta \beta_{S E P}$, the farther the orbit deviates from the $z$-static NKO, i.e. the wider the band covered on the cylindrical surface. Additionally, the larger the value for $\omega$, the narrower the band covered for a particular value for $\Delta \beta_{S E P}$. This has an interesting application as will be explained at the end of this chapter.

Finally, some of the orbits in Figure 10, e.g. the bottom right plot, again suggest the existence of periodic orbits. To find true periodic orbits for the displaced, cylindrical case, an approach very similar to the one described for the equatorial, cylindrical orbits on page 5 is used. Only now, since the orbit does not always cross the $(x, y)$-plane, the integration is not truncated upon crossing the $(x, y)$-plane, but after one full oscillating motion. The ratio of outof-plane and in-plane angular velocities then becomes: $\omega_{z} / \omega=\theta_{P} / 2 \pi$, which is used to establish the periodicity of the orbits. The result can be found in Figure 8, with some typical displaced, cylindrical orbits in Figure 11.

\subsection{Solar sail acceleration law}

The second type of acceleration that will be considered to generate cylindrical NKOs, is an ideal solar sail acceleration. An ideal solar sail is a sail that is perfectly reflecting. The incoming solar photons are therefore specularly reflected and the solar radiation pressure force acts perpendicular to the sail surface. Following (McInnes, 1999) and the definitions in Figure 1, the ideal solar sail acceleration can be written as:

$$
a=\beta_{s} \frac{\mu}{r^{2}} \cos ^{2} \alpha=\beta_{s} \bar{\omega}^{2} \rho\left(1+\left(\frac{z}{\rho}\right)^{2}\right)^{-1} \cos ^{2} \alpha
$$

with $\beta_{s}$ the sail lightness number, which is the ratio of the solar radiation pressure acceleration and the solar gravitational acceleration, or can equivalently be described as a function of the ratio of the spacecraft mass and the solar sail area (McInnes, 1999). Substituting this acceleration into Eq. 3, gives the required control law in implicit form:

$$
\begin{array}{r}
\cos (\alpha+\gamma) \cos ^{2} \alpha=\cos \left(\alpha+\tan ^{-1}\left(\frac{z}{\rho}\right)\right) \cos ^{2} \alpha= \\
\frac{1}{\beta_{s}}\left(1+\left(\frac{z}{\rho}\right)^{2}\right)\left(\left(1+\left(\frac{z}{\rho}\right)^{2}\right)^{-\frac{3}{2}}-\left(\frac{\omega}{\bar{\omega}}\right)^{2}\right)
\end{array}
$$

To solve Eq. 15 for $\cos \alpha$, the roots of the following sixth order polynomial need to be found: 

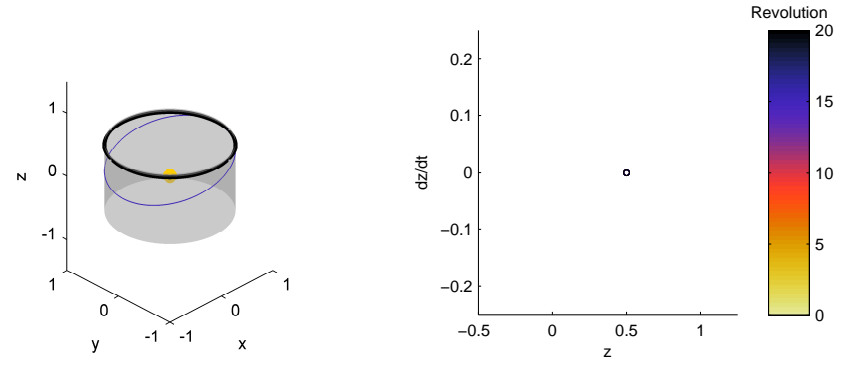

(a)
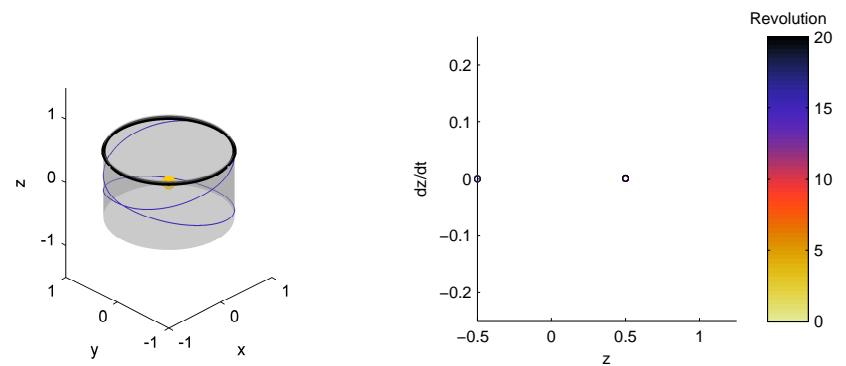

(b)
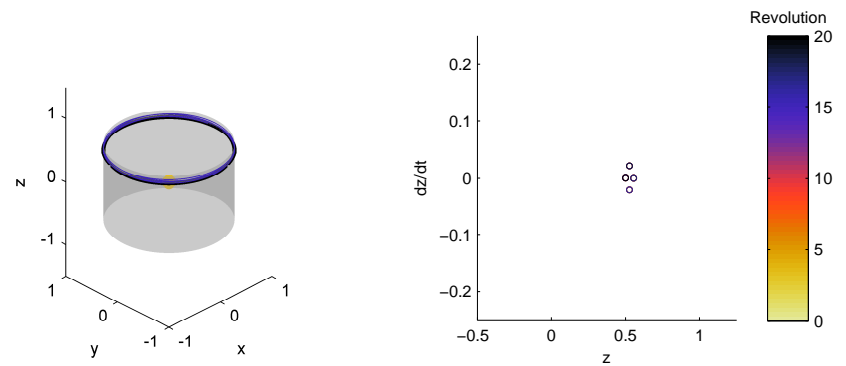

(c)
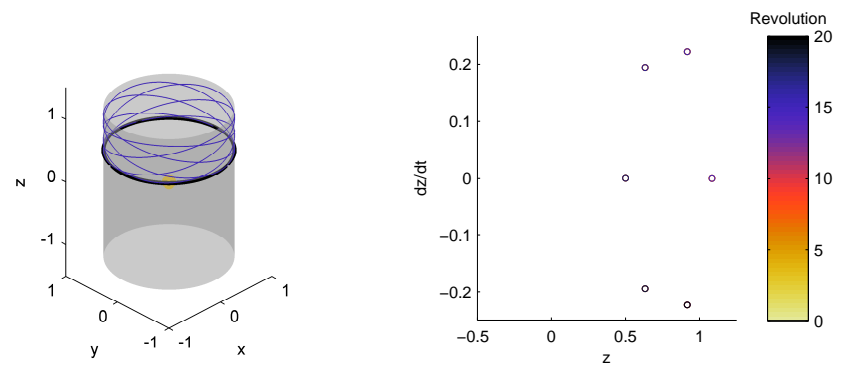

(d)

Figure 11: Periodic orbits (see black dots in Figure 8) and Poincaré maps for displaced, cylindrical NKOs with inverse-square acceleration law for $\rho=0.9$ and $z_{0}=0.5$. a) $\omega=1.058, \beta_{S E P}=0.283$. a) $\omega=1.020, \beta_{S E P}=0.296$. c) $\omega=0.915, \beta_{S E P}=0.508$. d) $\omega=0.850, \beta_{S E P}=0.703$. 


$$
x^{6}+\left(a^{2}-1\right) x^{4}-2 a b x^{3}+b^{2}=0
$$

with $x=\cos \alpha, a=\cos \gamma$ and $b=\frac{1}{\beta_{s}}\left(1+\left(\frac{z}{\rho}\right)^{2}\right)\left(\left(1+\left(\frac{z}{\rho}\right)^{2}\right)^{-\frac{3}{2}}-\left(\frac{\omega}{\bar{\omega}}\right)^{2}\right)$.

From Descartes rule of signs, it follows that this polynomial has a maximum of 2 positive and 2 negative real roots (Meserve, 1982). The negative roots can be discarded, since for a solar sail acceleration law, the constraint on $\cos \alpha$ is tightened (i.e. $\cos \alpha \geq 0$ ) due to the inability of a solar sail to generate an acceleration component in the direction of the Sun. Only a maximum of two positive real roots remain. However, since the original equation in Eq. 15 only has 3 real roots, only one of the two positive real roots remains as a true solution to Eq. 15.

\subsubsection{Family of displaced orbits}

For brevity, only the family of displaced, cylindrical orbits will be considered for the solar sail acceleration law. Especially, since by significantly decreasing $\beta_{s}$ with respect to the value for $\beta_{s, z-s t a t i c}$, the displaced orbits transform into equatorial orbits, as was already demonstrated in Figure 9 for the inverse square acceleration law. Only the plus-sign in Eq. 5 will thus be considered as well as $\alpha \geq 0$, i.e. the cone angle is either zero or away from the radial direction in counterclockwise direction. The condition $\alpha \geq 0$ is automatically satisfied when evaluating $\cos ^{-1} x$ since the information on the sign of $\alpha$ is not contained in $x$. Evaluating $\cos ^{-1} x$ will therefore by default return positive values for $\alpha$. Finally, the constraints $-1 \leq \cos (\alpha+\gamma) \leq 1$ need to be taken into account.

Due to the lack in an explicit expression for $\cos \alpha$ (and thus also for $\cos (\alpha+\gamma)$ ), the constraints on $\cos (\alpha+\gamma)$ cannot be expressed as an analytical formulation for the maximum allowed in-plane angular velocity and the minimum required sail lightness number. These constraints can therefore only be enforced within the integration of motion, leading to a truncation of the integration when either of the constraints is violated.

The results, in terms of the region of feasibility for displaced, solar sail cylindrical orbits, is shown in Figure 12, again for the test case $\rho=0.9$ and $z_{0}=0.5$ and with the thick red line dividing the feasibility region indicating the lightness number, $\beta_{s}$, required to maintain the $z$-static NKO (McInnes, 1999):

$$
\beta_{s, z-\text { static }}=\frac{\left(1+\left(\frac{z_{0}}{\rho}\right)^{2}\right)^{\frac{1}{2}}\left(\left(\frac{z_{0}}{\rho}\right)^{2}+\left(1-\left(\frac{\omega}{\bar{\omega}_{r}}\right)^{2}\right)^{2}\right)^{\frac{3}{2}}}{\left(\left(\frac{z_{0}}{\rho}\right)^{2}+\left(1-\left(\frac{\omega}{\bar{\omega}_{r}}\right)^{2}\right)\right)^{2}}
$$

with $\bar{\omega}_{r}=\sqrt{\frac{\mu}{r^{3}}}$ and $r=\sqrt{\rho^{2}+z^{2}}$. Note that the sharp edge on the right side of the feasibility region is very close to the maximum value for the in-plane angular velocity for which the $z$-static NKO still exists, $\omega_{z \text {-static,max }}$. This limit is given by (McInnes, 1999) as: 


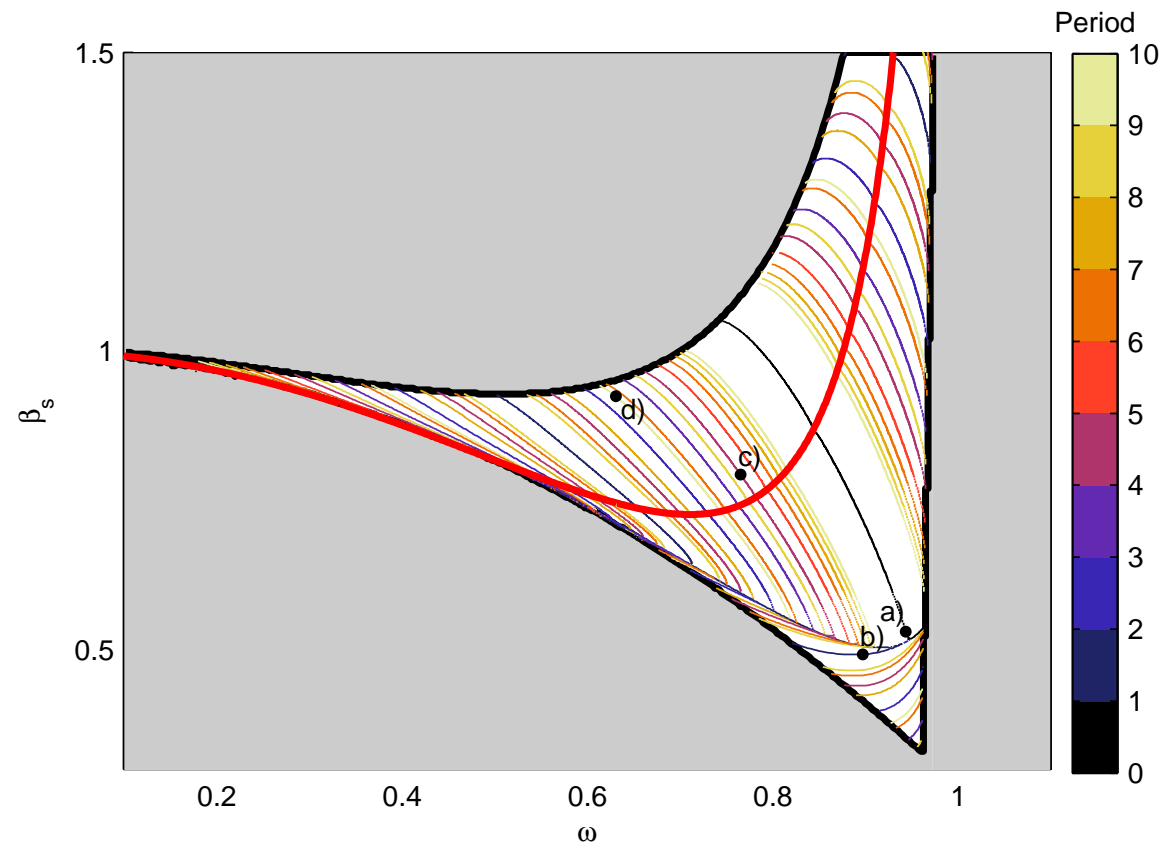

Figure 12: Feasibility region including periodic orbits for displaced, cylindrical NKOs with solar sail acceleration law for $\rho=0.9$ and $z_{0}=0.5$. The thick red line dividing the feasibility region equals Eq. 17. The black dots correspond to the orbits in Figure 13.

$$
\omega_{z-\text { static, } \max } \leq \sqrt{\frac{\mu}{r \rho^{2}}}
$$

Close to this limit, the required value for $\beta_{s, z-s t a t i c}$ increases greatly, as the thick red line in Figure 12 shows, and only lightness numbers close to these large values for $\beta_{s, z-s t a t i c}$ provide feasible cylindrical orbits, which far exceed the interval considered for $\beta_{s}$ in Figure 12 .

Finally, the same analysis as for the displaced, cylindrical orbits with an inverse square law is applied to find true periodic orbits for the solar sail case. The result is included in Figure 12 with some typical solar sail periodic orbits in Figure 13.

\subsubsection{Analysis in the $\left(\rho, z_{0}\right)$-domain}

The analyses in the previous subsections have assumed one particular combination of the projected radius, $\rho$, and the initial out-of-plane displacement, $z_{0}$, and shown the feasibility and periodicity of orbits in the $\left(\omega, \beta_{S E P}\right)-$ or $\left(\omega, \beta_{s}\right)$ domain. The reason for choosing $\omega$ and $\beta$ as design parameters is the fact that, 

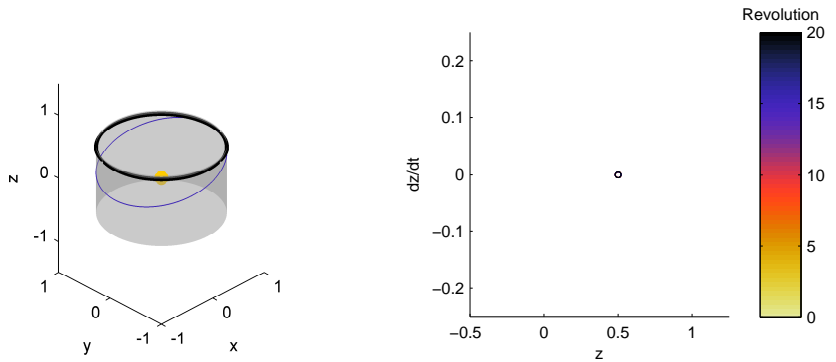

(a)
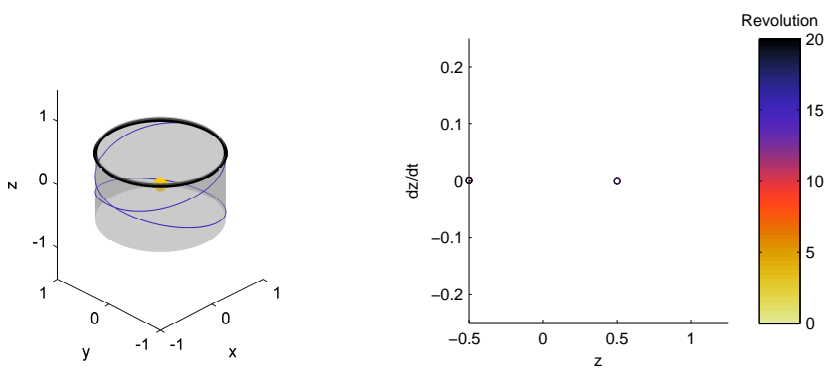

(b)
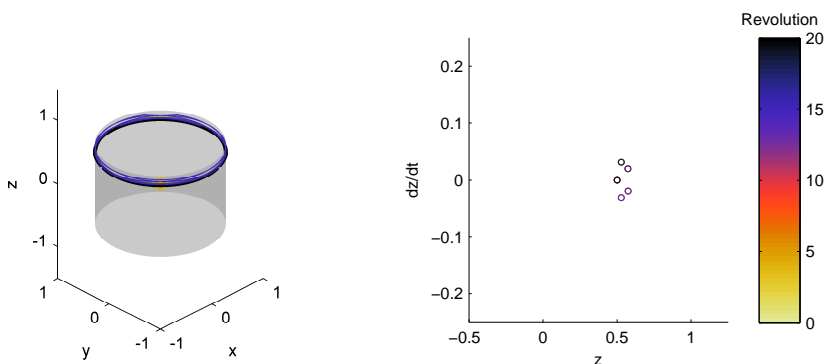

(c)
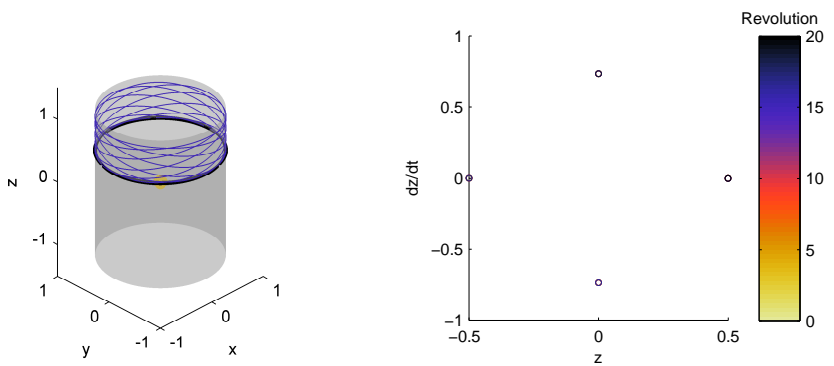

(d)

Figure 13: Periodic orbits (see black dots in Figure 12) and Poincaré maps for displaced, cylindrical NKOs with solar sail acceleration law for $\rho=0.9$ and $z_{0}=0.5$. a) $\omega=0.943, \beta_{s}=0.530$. b) $\omega=0.897, \beta_{s}=0.492$. c) $\omega=0.765$, $\beta_{s}=0.793$. d) $\omega=0.631, \beta_{s}=0.924$. 
to a certain extent, the constraints and feasibility can be derived analytically. However, for mission design purposes, it might also be of interest to show periodic orbits in the $\left(\rho, z_{0}\right)$-domain. Then, it becomes immediately clear where periodic orbits exist with a particular in-plane angular velocity, $\omega$, and propulsion technology, $\beta_{S E P}$ or $\beta_{s}$. The result of such an analysis is shown in Figure 14. To limit the amount of results provided, only the solar sail case is considered and a hypothetical value for the lightness number of $\beta_{s}=0.5$ is selected. Furthermore, the results are created for $\omega=1$, equalling the angular velocity of the Earth around the Sun. Finally, the thick red line dividing the feasibility region once again represents the $z$-static NKOs. The plot now clearly indicates where periodic, cylindrical orbits exist that are synchronous with the Earth and are enabled with a propulsion technology of $\beta_{s}=0.5$. These results in combination with the results presented in Figure 12 provide a complete overview of the possible solar sail, displaced, cylindrically constrained non-Keplerian orbits.

A final consideration is devoted to the sensitivity in the initial conditions of the periodic orbits. The figures throughout this chapter have demonstrated clusters of periodic orbits in the $(\omega, \beta)$ - and $\left(\rho, z_{0}\right)$-domains and in some particular cases even bifurcations where a line representing orbits with one particular period branches of a line representing orbits with a different period. While an example of the latter will be given in Chapter 2, Figure 15 zooms in on an area of Figure 14 where the lines are heavily clustered. Although the difference in $\rho$ and $z_{0}$ between the three periodic orbits is only $0.3 \%$ and $4.3 \%$ at maximum, respectively, three completely different orbits with periods of 7,9 and 2 exist within that small domain. In these cases, precise insertion into the orbit as well as close navigation during the orbit will be essential to guarantee that the sail will stay on the targeted orbit.

\subsection{Applications}

A range of applications exist for the orbits presented throughout this chapter. Generally, the same applications that were mentioned for the $z$-static NKOs in the introduction of this paper also hold for the cylindrical orbits but at a reduced required performance of the propulsion system: by definition, the family of south orbits require a lower propulsion performance than the $z$-static NKO from which they bifurcate (i.e. $\beta_{S E P}<\beta_{S E P, z-\text { static }}$ and $\beta_{s}<\beta_{s, z-s t a t i c}$ ) and some of these orbits remain very close to the $z$-static NKO. A good example is given in the top right plot of Figure 10, which allows for a $15 \%$ reduction in the required scaling factor while remaining close to the $z$-static NKO. This advantage occurs mainly for large values for the in-plane angular velocity, see the phase spaces in Figure 9, where some of the darks lines on the left of the grey transparent plane (for which $\beta<\beta_{z-\text { static }}$ ) remain close to the dots on this grey transparent plane that represent the $z$-static NKOs. Therefore, if a small deviation from the $z$-static NKO is allowed, particular cylindrical NKOs could reduce the required propulsion system performance compared to $z$-static NKOs. More specifically, the cylindrical orbits allow to hover above the ecliptic plane or above any of the planetary orbital planes. This provides a way to view the 


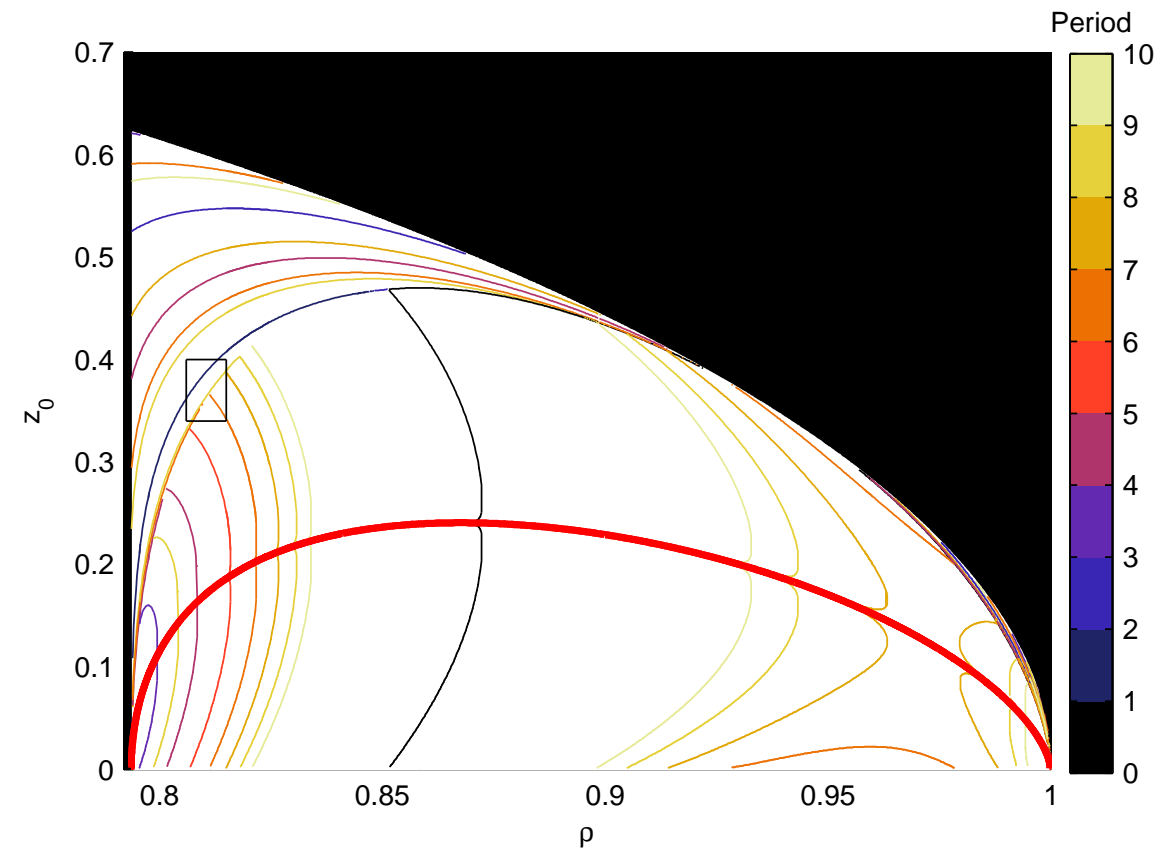

Figure 14: Feasibility region and periodic orbits in the $\left(\rho, z_{0}\right)$-domain for displaced, cylindrical NKOs with solar sail acceleration law for $\omega=1.0$ and $\beta_{s}=0.5$. The thick red line dividing the feasibility region equals Eq. 17 . The black box refers to the detail in Figure 15.

Sun from out of the ecliptic or, by choosing the correct angular velocity such that the cylindrical NKO is synchronous with any of the inner Solar System planets, to view the planets from out of their orbital plane.

\section{Spherically constrained NKOs}

The approach to find spherically constrained NKOs is very similar to the one taken to find cylindrically constrained orbits. However, now the equations of motion are considered in a spherical coordinate system $(r, \theta, \phi)$ as shown in Figure 16. Again, assuming a central gravitational force field and an acceleration in the $(r, \phi)$-plane only, the equations of motion can be written as:

$$
\begin{aligned}
\ddot{r}-r \dot{\theta}^{2} \cos ^{2} \phi-r \dot{\phi}^{2} & =-\frac{\mu}{r^{2}}+a \cos \alpha \\
r \cos \phi \ddot{\theta}+2 \dot{r} \dot{\theta} \cos \phi-2 r \dot{\theta} \dot{\phi} \sin \phi & =0 \\
r \ddot{\phi}+2 \dot{\phi} \dot{\phi}+r \dot{\theta}^{2} \sin \phi \cos \phi & = \pm a \sin \alpha
\end{aligned}
$$

and to remain on a spherical surface, a constraint on the radius $r$ is introduced:

$$
r=\text { constant } \rightarrow \ddot{r}(t)=\dot{r}(t)=0
$$




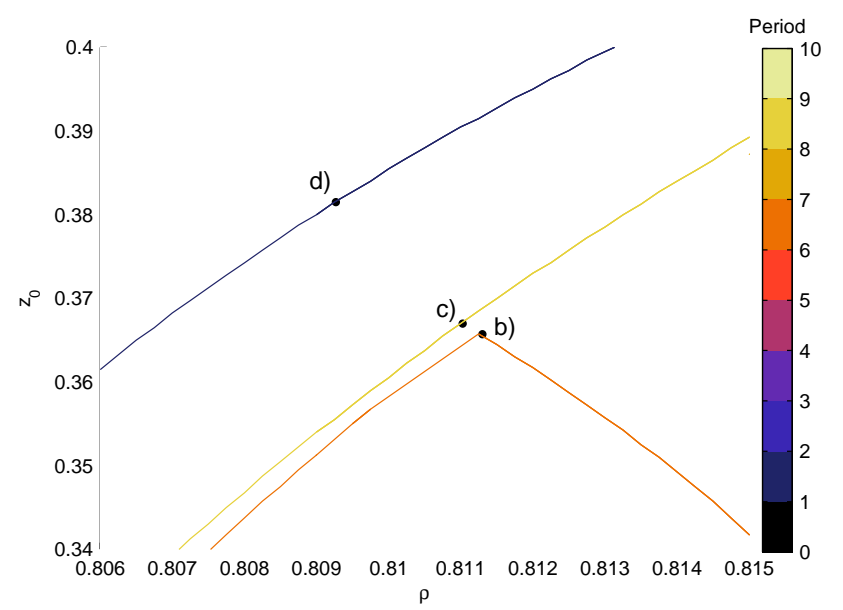

(a)
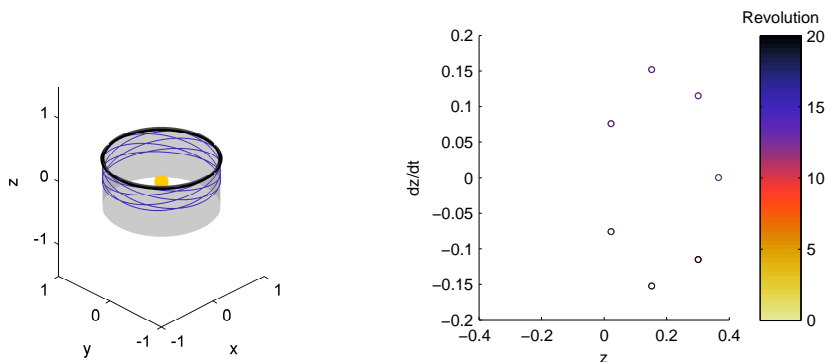

(b)
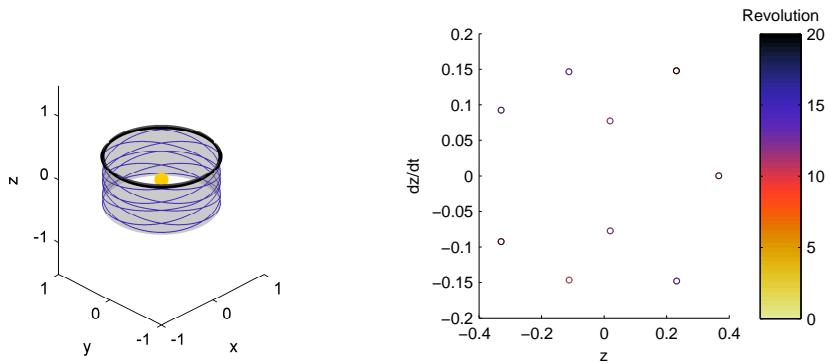

(c)
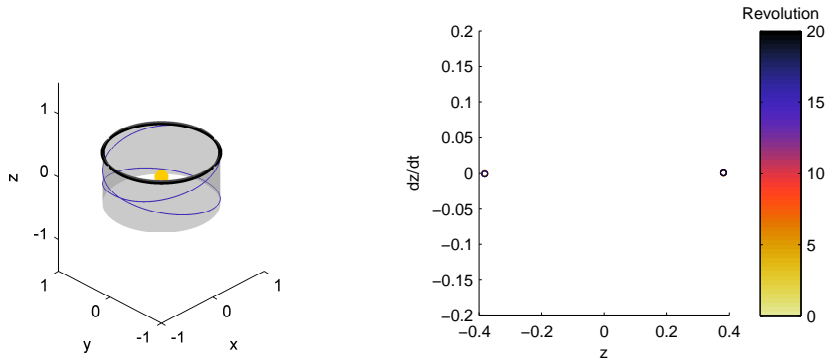

(d)

22

Figure 15: a) Detail of Figure 14. The black dots correspond to the orbits in subplots b-d). b) $\rho=0.8114, z_{0}=0.3657$. c) $\rho=0.8110, z_{0}=0.3670$. d) $\rho=0.8093, z_{0}=0.3815$. 


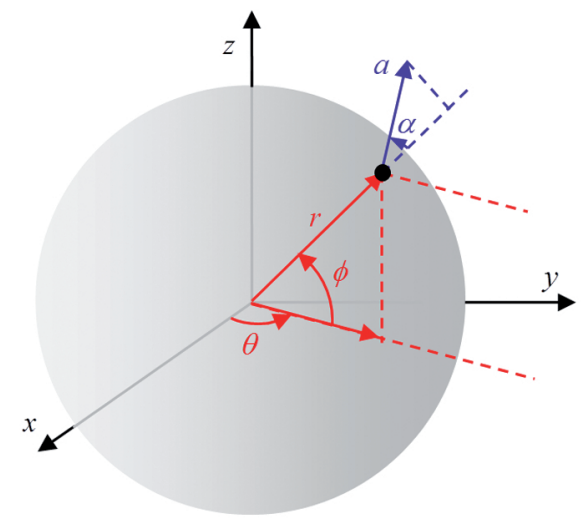

Figure 16: Definition of spherical reference frame.

A parameter similar to $\bar{\omega}$ as used for the cylindrical case is defined: $\bar{\omega}_{r}=$ $\sqrt{\mu / r^{3}}$, which now equals the angular velocity of a Keplerian orbit with radius $r$. The equations of motion then reduce to:

$$
\begin{gathered}
\cos \alpha=\frac{r}{a}\left(\bar{\omega}_{r}^{2}-\dot{\theta}^{2} \cos ^{2} \phi-\dot{\phi}^{2}\right) \\
\ddot{\theta}=2 \dot{\theta} \dot{\phi} \tan \phi \\
\ddot{\phi}=-\dot{\theta}^{2} \sin \phi \cos \phi \pm \frac{a}{r} \sin \alpha
\end{gathered}
$$

Equation 21 once again provides the required control law; equation 22 shows that, contrary to the cylindrical case, the in-plane angular velocity, $\dot{\theta}$, is not constant; and finally, with initial conditions $\phi(0)=\phi_{0}, \dot{\phi}(0)=0$, Eq. 23 again resembles a highly non-linear oscillator and a \pm -minus sign is included in front of the acceleration term to generate the two families of orbits as discussed for the cylindrical case: equatorial orbits (for the minus-sign) and displaced orbits (for the plus-sign).

\subsection{Inverse square acceleration law}

Using the new definition for $\bar{\omega}_{r}$, the inverse-square acceleration law can now be written as:

$$
a=\beta_{S E P} \frac{\mu}{r^{2}}=\beta_{S E P} \bar{\omega}_{r}^{2} r
$$

which is constant on the spherical surface and reduces the equations of motion to:

$$
\begin{aligned}
\cos \alpha & =\frac{1}{\beta_{S E P}}\left(1-\frac{1}{\bar{\omega}_{r}^{2}}\left(\dot{\theta}^{2} \cos ^{2} \phi+\dot{\phi}^{2}\right)\right) \\
\ddot{\theta} & =2 \dot{\theta} \dot{\phi} \tan \phi \\
\ddot{\phi} & =-\dot{\theta}^{2} \sin \phi \cos \phi \pm \beta_{S E P} \bar{\omega}_{r}^{2} \sin \alpha
\end{aligned}
$$


Again, feasible spherical orbits are found only if $-1 \leq \cos \alpha \leq 1$, which can be translated into the following constraints on the in-plane angular velocity, $\dot{\theta}(t)$ and the scaling factor, $\beta_{S E P}$ :

$$
\begin{gathered}
\dot{\theta}(t) \leq \sqrt{\frac{\bar{\omega}_{r}^{2}\left(1+\beta_{S E P}\right)-\dot{\phi}^{2}}{\cos ^{2} \phi}} \\
\beta_{S E P} \geq 1-\frac{1}{\bar{\omega}_{r}^{2}}\left(\dot{\theta}^{2} \cos ^{2} \phi+\dot{\phi}^{2}\right)
\end{gathered}
$$

Due to the fact that the in-plane angular velocity is not constant throughout the orbit, the evaluation of the constraints in Eqs. 26 and 27 is less straight forward than for the cylindrical case. Especially Eq. 26 is difficult to evaluate analytically since both the left-hand side and right-hand side change along the orbit. General constraints as derived in Eqs. 7 and 8 for the cylindrical case can therefore not be obtained. Instead, the evaluation of the constraints will be investigated for each family of spherical NKOs separately, first for the equatorial orbits and subsequently for the displaced orbits.

\subsubsection{Equatorial orbits}

For equatorial orbits, analyses have shown that the maximum value of the righthand side of Eq. 27 occurs at the initial condition, i.e. at $\phi=\phi_{0}$ and $\dot{\phi}=0$. Therefore, the minimum value for the scaling factor is given by:

$$
\beta_{S E P} \geq \beta_{S E P, \min }=1-\frac{\dot{\theta}_{0}^{2}}{\bar{\omega}_{r}^{2}} \cos ^{2} \phi_{0}
$$

and can be evaluated analytically. Instead, the minimum value of both sides of Eq. 26 occur upon crossing the $(x, y)$-plane, i.e. at $\phi=0$, leading to the following constraint on the in-plane angular velocity:

$$
\dot{\theta}_{\phi=0} \leq \dot{\theta}_{\phi=0, \max }=\sqrt{\bar{\omega}_{r}^{2}\left(1+\beta_{S E P}\right)-\dot{\phi}_{\phi=0}^{2}}
$$

Since both $\dot{\theta}_{\phi=0}$ and $\dot{\phi}_{\phi=0}$ cannot be known a priori, the constraint in Eq. 29 can only be enforced numerically by integrating the equations of motion and truncating the integration as soon as the constraint is violated. The result, in terms of the feasibility region for equatorial, spherical NKOs is shown in Figure 17a for the same case as in Section 1: $\rho_{0}=0.9$ and $z_{0}=0.5$ (i.e. $r=1.03$ and $\left.\phi_{0}=0.16 \pi\right)$. Since the in-plane angular velocity is not constant, the design parameter on the $x$-axis is the initial angular velocity, $\dot{\theta}_{0}$. Interesting to note is the case where $\beta_{S E P}=0$ and $\dot{\theta}_{0}=1.095$, which represents the circular Keplerian orbit that lies on the spherical surface.

A first impression of the types of equatorial, spherically constrained NKOs that are feasible is provided in Figure 17b) for two particular values of the scaling factor. The figures again clearly show the oscillating behavior of these orbits around the $(x, y)$-plane and hint at the possibility of finding periodic orbits. 


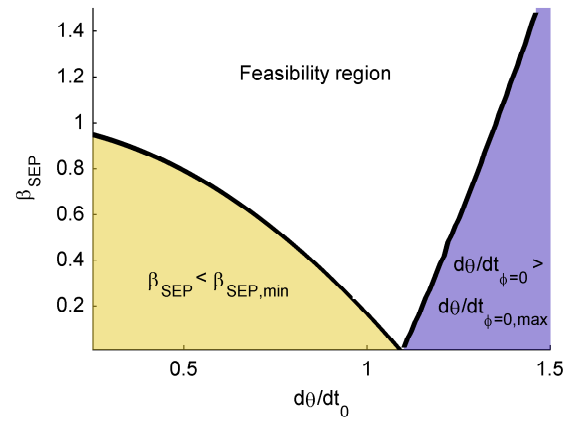

(a)
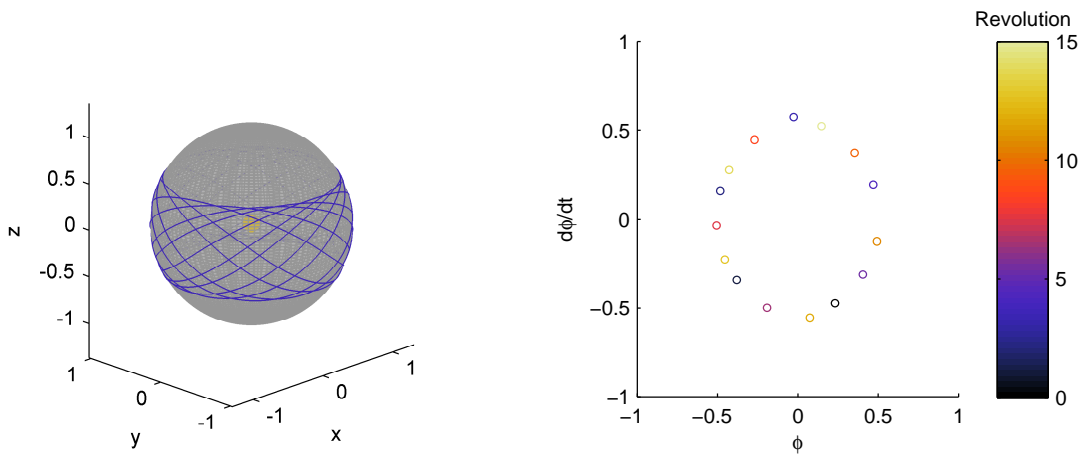

(b)
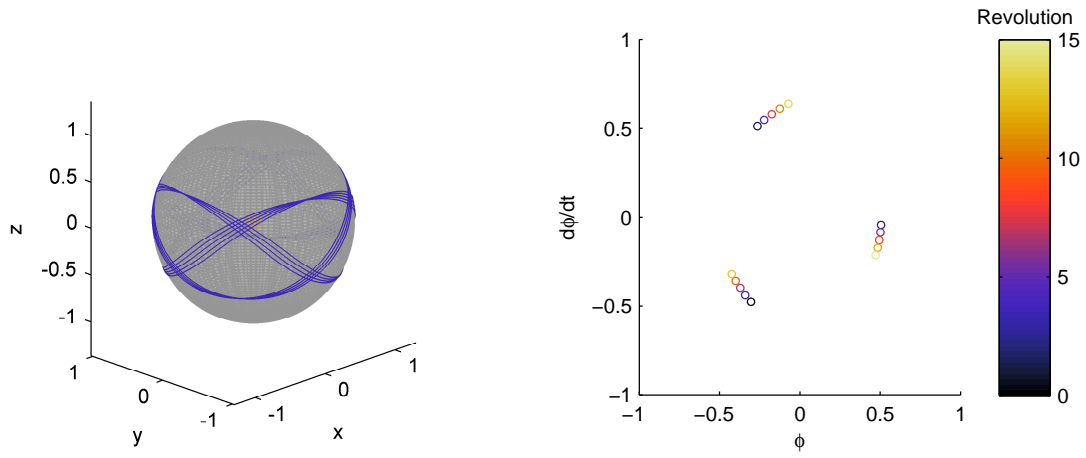

(c)

Figure 17: Equatorial, spherical NKOs with inverse-square acceleration law for $\rho_{0}=0.9$ and $z_{0}=0.5$. a) Feasibility region. b) Orbits and Poincaré maps for $d \theta / d t_{0}=1, \beta_{S E P}=0.2$ (top) and $\beta_{S E P}=0.3$ (bottom). 
True periodic orbits are once again sought for using an approach very similar to the one used for equatorial, cylindrical orbits: the orbit is integrated forward from its initial conditions until crossing the $(x, y)$-plane, which occurs at $\theta=$ $\theta_{\phi=0}$. A full period of the orbit, $P$, would thus equal $P=4 \theta_{\phi=0}$, which can be expressed as a fraction of a full revolution as $4 \theta_{\phi=0} / 2 \pi$ and equals the ratio of out-of-plane and in-plane periods: $P_{\text {out }} / P_{\text {in }}=4 \theta_{\phi=0} / 2 \pi$. Subsequent steps in the approach are the same as for the cylindrical case: if the $P_{\text {out }} / P_{i n}$-fraction equals an irreducible fraction (e.g. $\frac{1}{2}, \frac{2}{3}, \frac{4}{5}$, etc.) the orbit is periodic, where the period is given by the nominator of the irreducible fraction. A fine grid search over values for $\dot{\theta}_{0}$ and $\beta_{S E P}$ provides the periodic equatorial, spherical orbits as shown in Figure 18, with some example periodic orbits in Figure 19.

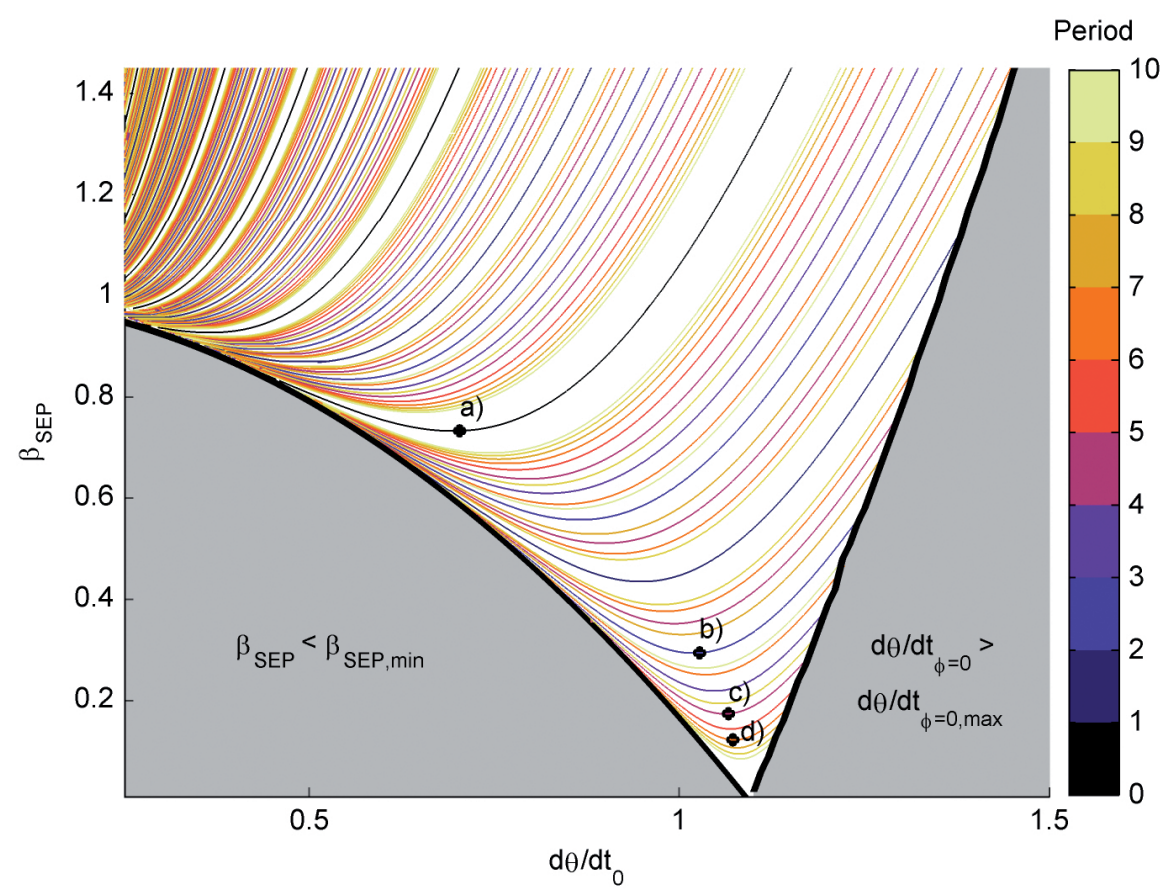

Figure 18: Periodic equatorial, spherical NKOs with inverse-square acceleration law for $\rho_{0}=0.9$ and $z_{0}=0.5$. The black dots correspond to the orbits in Figure 19.

\subsubsection{Displaced orbits}

When considering the plus-sign in Eq. 23, displaced, spherical NKOs can be found. As for the cylindrical case, the analyses start from the $z$-static NKOs which are part of the set of spherical NKOs. The required scaling factor for these $z$-static NKOs, $\beta_{S E P, z-s t a t i c}$, can be found by setting $\ddot{\phi}(t)=\dot{\phi}(t)=0$ in 

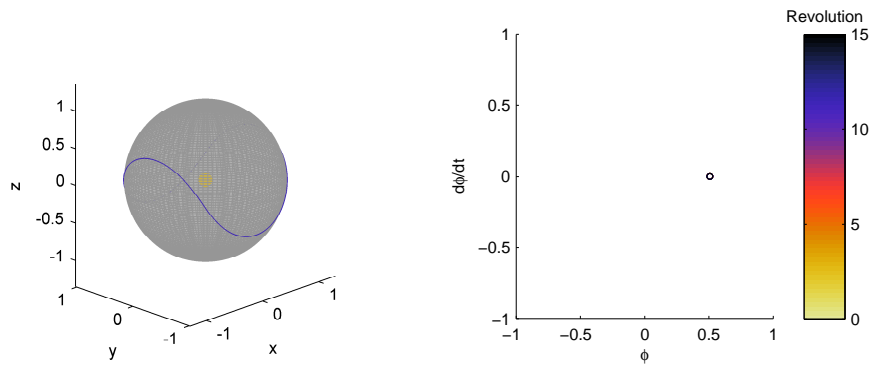

(a)
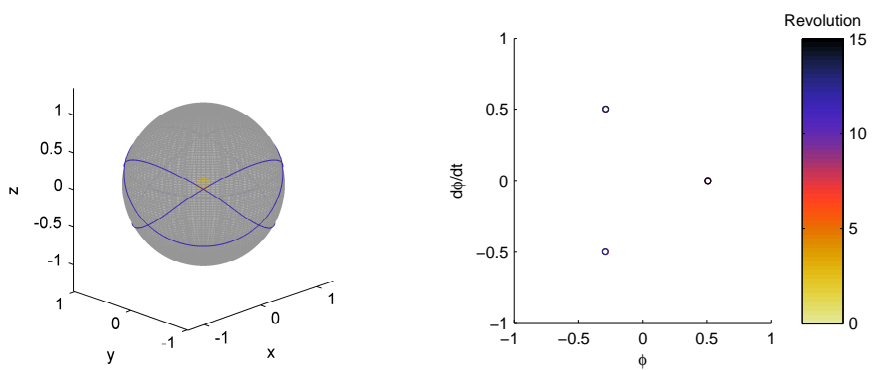

(b)
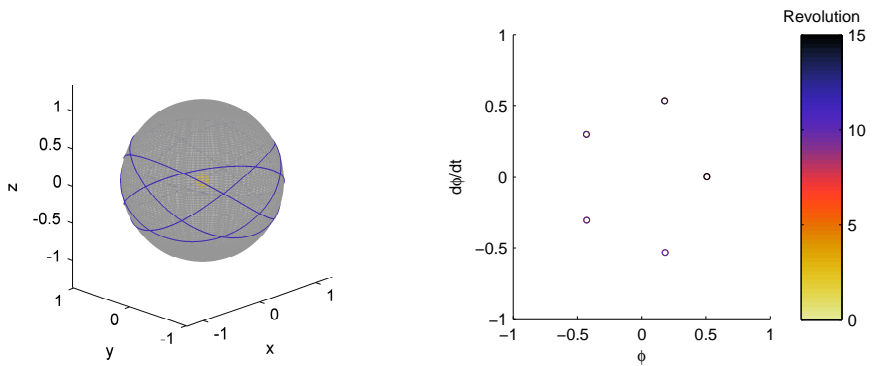

(c)
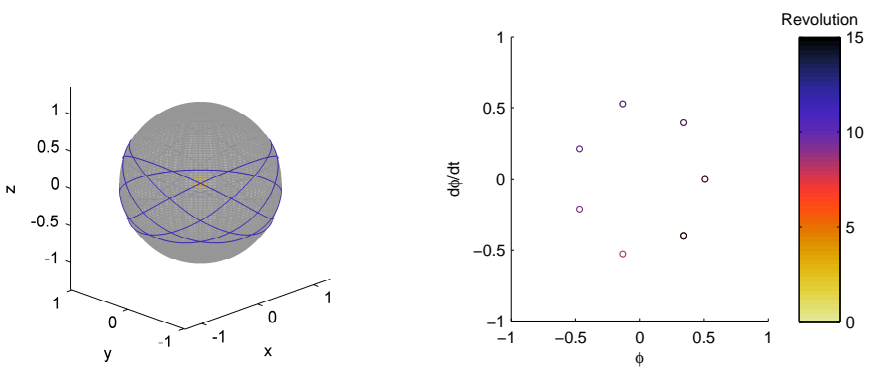

(d)

Figure 19: Periodic orbits (see black dots in Figure 18) and Poincaré maps for equatorial, spherical NKOs with inverse-square acceleration law for $\rho_{0}=0.9$ and $z_{0}=0.5$. a) $d \theta / d t_{0}=0.703, \beta_{S E P}=0.733$. b) $d \theta / d t_{0}=1.027, \beta_{S E P}=0.295$. c) $\left.d \theta / d t_{0}=1.066, \beta_{S E P}=0.175 . \mathrm{d}\right) . d \theta / d t_{0}=1.072, \beta_{S E P}=0.123$. 
Eq. 25:

$$
\beta_{S E P, z-\text { static }}=\sqrt{\left(\left(\frac{\omega}{\bar{\omega}_{r}}\right)^{2} \sin \phi \cos \phi\right)^{2}+\left(1-\left(\frac{\omega}{\bar{\omega}_{r}}\right)^{2} \cos ^{2} \phi\right)^{2}}
$$

Note that, for the $z$-static NKOs, the in-plane angular velocity is constant and can once again be denoted by $\omega$. Furthermore, when considering the difference in definition between $\bar{\omega}$ and $\bar{\omega}_{r}$, Eq. 30 can be shown to equal Eq. 11.

Again, by deviating from $\beta_{S E P, z-s t a t i c}$, additional spherically confined orbits can be found: the sub-family of 'north orbits' are created by increasing $\beta_{S E P}$ with respect to $\beta_{S E P, z-\text { static }}$, while the sub-family of 'south orbits' are created by decreasing $\beta_{S E P}$ with respect to $\beta_{S E P, z-\text { static. }}$. The evaluation of the constraints in Eqs. 26 and 27 is different for each type of orbit, but is very similar to the cylindrical case, as will be discussed below.

To obtain the minimum required value for $\beta_{S E P}$, the constraint in Eq. 27 needs to be evaluated at $\phi_{\min }$, which is known for north orbits $\left(\phi_{\min }=\phi_{0}\right)$ but unknown for south orbits. The constraint on $\beta_{S E P}$ can therefore only be determined analytically for north orbits and is evaluated numerically for south orbits:

$$
\beta_{S E P, \text { north }} \geq \beta_{S E P, \text { min }}=1-\frac{\dot{\theta}_{0}^{2}}{\bar{\omega}_{r}^{2}} \cos ^{2} \phi_{0}
$$

Furthermore, it appears that the constraint on $\dot{\theta}(t)$ can be evaluated at $\phi_{\max }$, which is known for south orbits $\phi_{\max }=\phi_{0}$, but is unknown for north orbits. For south orbits, the constraint on $\dot{\theta}(t)$ can therefore be determined analytically through:

$$
\dot{\theta}_{0, \text { south }} \leq \dot{\theta}_{0, \max }=\frac{\bar{\omega}_{r} \sqrt{\left(1+\beta_{S E P}\right)}}{\cos \phi_{0}}
$$

The resulting feasibility region is shown in Figure 20, which includes the results of the periodicity analysis, and is shown as the system's phase space in Figure 21 (again for the case $\rho_{0}=0.9$ and $z_{0}=0.5$ ). In Figure 20, the constraint in Eq. 31 is indicated with a dashed white line (again indicating that $z$-static NKOs are feasible for all $\dot{\theta}_{0}$ ), while the constraint in Eq. 32 is shown with a dotted white line. Although the figure seems to suggest that, for north orbits, no limit exists on the allowable value for $\beta_{S E P}$, such a limit does exist, but only for extremely large values for $\beta_{S E P}$. Both figures again clearly demonstrate the existence of north and south orbits and - in some particular cases - show the transition of displaced orbits into equatorial orbits for small enough values of $\beta_{S E P}$. Finally, some example periodic orbits are provided in Figure 22.

\subsection{Solar sail acceleration law}

The final case that will be investigated considers the displaced, spherical orbits with a solar sail acceleration law. Using the new definition for $\bar{\omega}_{r}$, the solar sail acceleration law can now be written as:

$$
a=\beta_{s} \frac{\mu}{r^{2}} \cos ^{2} \alpha=\beta_{s} \bar{\omega}_{r}^{2} r \cos ^{2} \alpha
$$




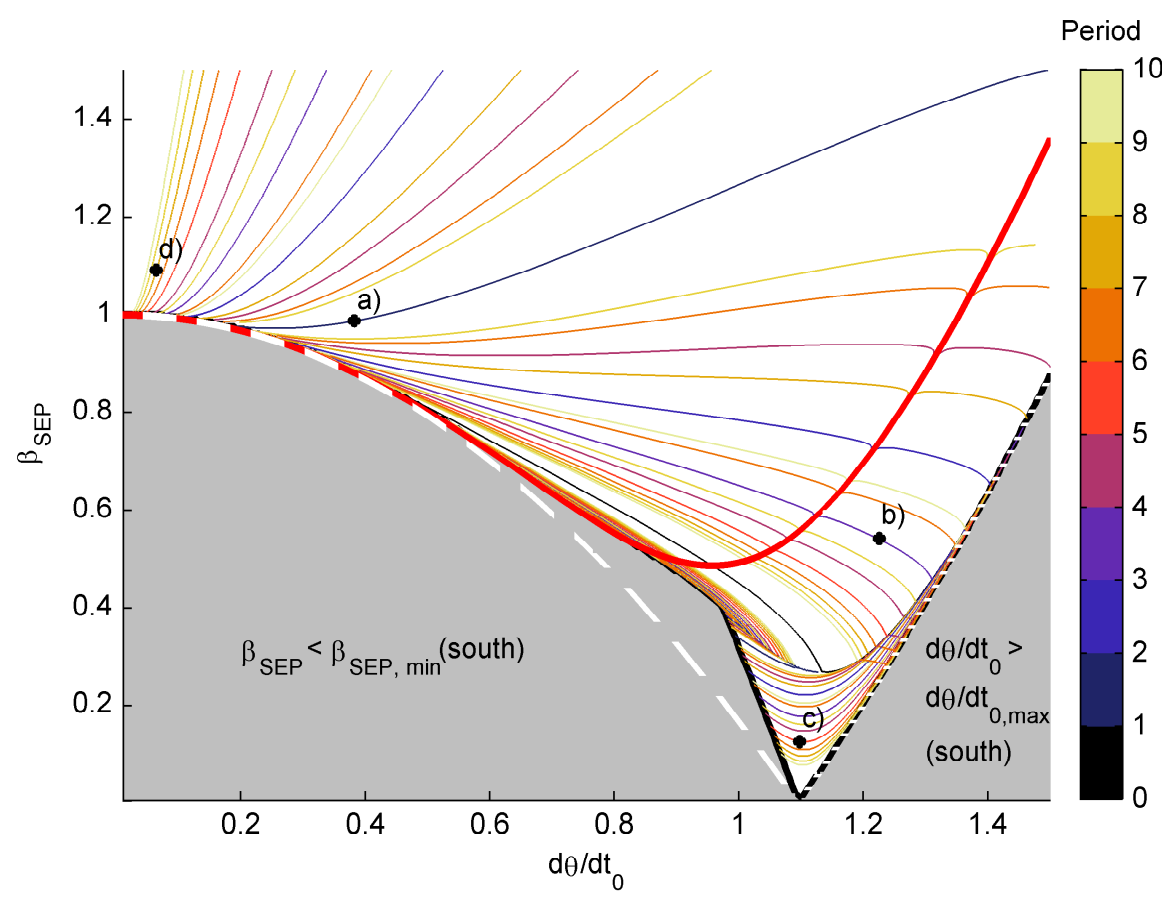

Figure 20: Feasibility region including periodic orbits for displaced, spherical NKOs with inverse-square acceleration law for $\rho_{0}=0.9$ and $z_{0}=0.5$. The thick red line dividing the feasibility region equals Eq. 30. The dashed white line equals Eq. 31, the dotted white line equals Eq. 32. The black dots correspond to the orbits in Figure 22.

which reduces the equations of motion in Eqs. 21-23 to:

$$
\begin{aligned}
\cos \alpha & =\sqrt[3]{\frac{1}{\beta_{s}}\left(1-\frac{1}{\omega_{r}^{2}}\left(\dot{\theta}^{2} \cos ^{2} \phi+\dot{\phi}^{2}\right)\right)} \\
\ddot{\theta} & =2 \dot{\theta} \dot{\phi} \tan \phi \\
\ddot{\phi} & =-\dot{\theta}^{2} \sin \phi \cos \phi+\beta_{s} \bar{\omega}_{r}^{2} \cos ^{2} \alpha \sin \alpha
\end{aligned}
$$

As for the cylindrically constrained orbits, only the family of displaced, spherical NKOs will be considered for brevity.

\subsubsection{Family of displaced orbits}

The feasibility region can once again be derived from the constraint $0 \leq \cos \alpha \leq$ 1 where the lower limit takes into account the inability of the solar sail to generate a component of the acceleration in the direction of the Sun. This lower limit results in a maximum value for the in-plane angular velocity for south 


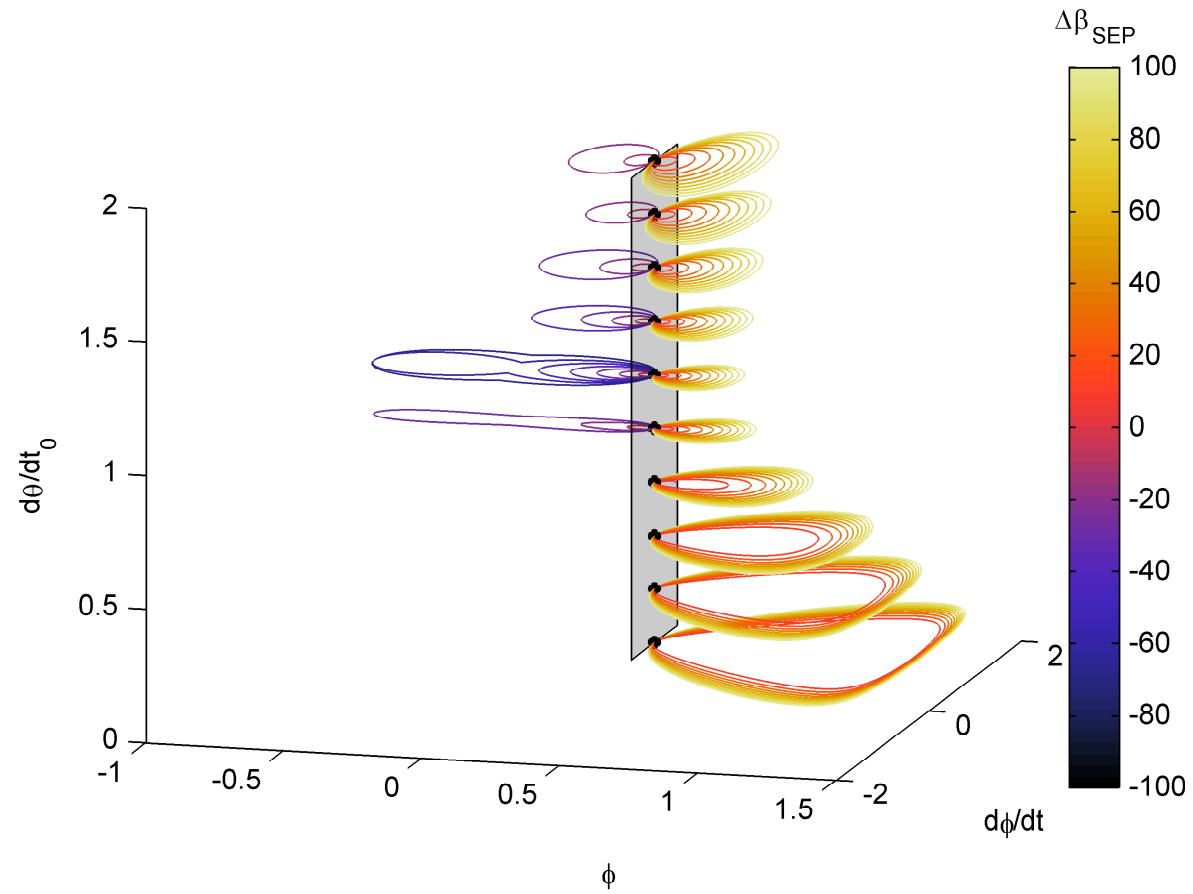

Figure 21: Phase spaces for displaced, spherical NKOs with inverse-square acceleration law for $\rho_{0}=0.9$ and $z_{0}=0.5$. Colors indicate percentage deviation from $\beta_{S E P, z-\text { static }}$ with a step size of $10 \%$.

orbits that is very similar to the constraint in Eq. 32:

$$
\dot{\theta}_{0, \text { south }} \leq \dot{\theta}_{0, \max }=\frac{\bar{\omega}_{r}}{\cos \phi_{0}}
$$

This constraint is independent of the value for $\beta_{s}$ and equals the maximum allowable in-plane angular velocity for a solar sail $z$-static NKO, see Eq. 18, as well as the in-plane angular velocity of the Keplerian orbit that lies on the spherical surface (as discussed on page 24).

Investigating the upper limit on $\cos \alpha$, it appears that for north orbits the same constraint applies as in Eq. 31 for an inverse-square acceleration law:

$$
\beta_{s, \text { north }} \geq \beta_{s, \text { min }}=1-\frac{\dot{\theta}_{0}^{2}}{\bar{\omega}_{r}^{2}} \cos ^{2} \phi_{0}
$$

The feasibility region in Figure 23 clearly indicates the constraints in Eqs. 35 and 36 with dotted and dashed white lines, respectively. Note that the constraints on the minimum required lightness number for south orbits and the maximum initial in-plane angular velocity for north orbits can once again only be determined numerically. The latter constraint limits the maximum achievable 

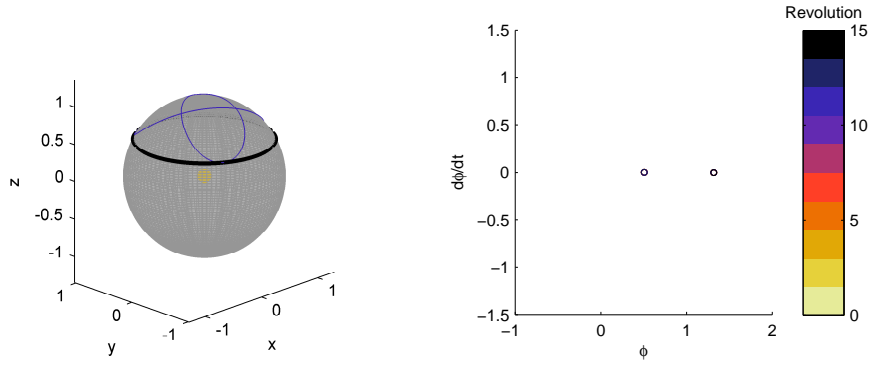

(a)
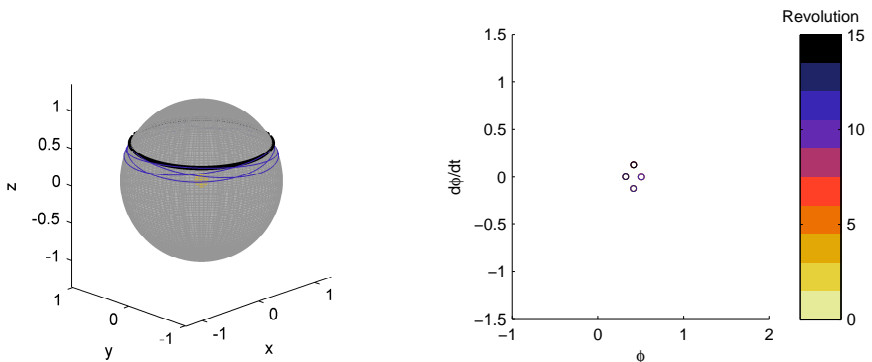

(b)
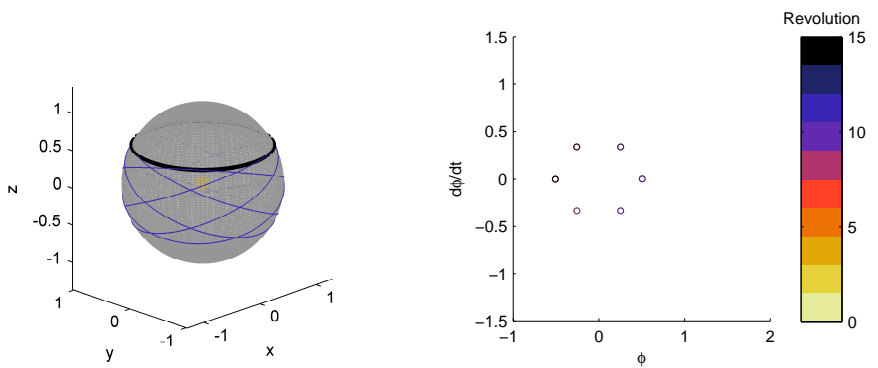

(c)
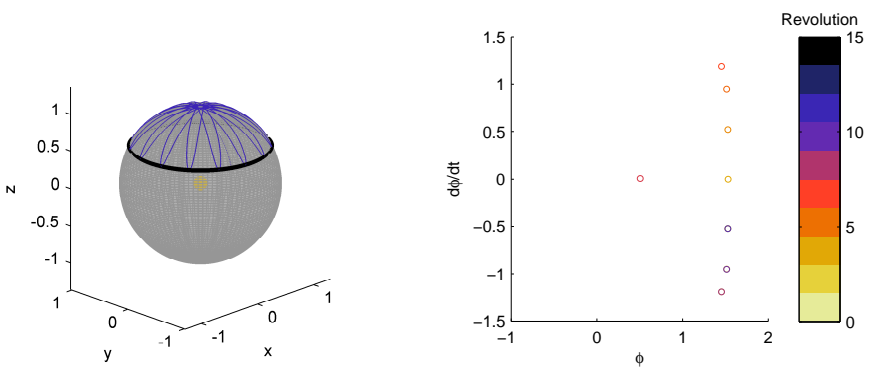

(d)

Figure 22: Periodic orbits (see black dots in Figure 20) and Poincaré maps for displaced, spherical NKOs with inverse-square acceleration law for $\rho_{0}=0.9$ and $z_{0}=0.5$. a) $d \theta / d t_{0}=0.382, \beta_{S E P}=0.987$. b) $d \theta / d t_{0}=1.225, \beta_{S E P}=0.542$. c) $\left.d \theta / d t_{0}=1.097, \beta_{S E P}=0.126 . \mathrm{d}\right) . d \theta / d t_{0}=0.064, \beta_{S E P}=1.091$. 
lightness number for north orbits, although Figure 23 might give the impression that the lightness number can be increased unlimitedly. Furthermore, the thick red line dividing the feasiblity region indicates the lightness number required to maintain the solar sail on a $z$-static NKO. Finally, using the exact same approach as for the other spherical orbits, periodic displaced, solar sail spherical orbits can be found, see Figure 23, with some example periodic orbits in Figure 24.

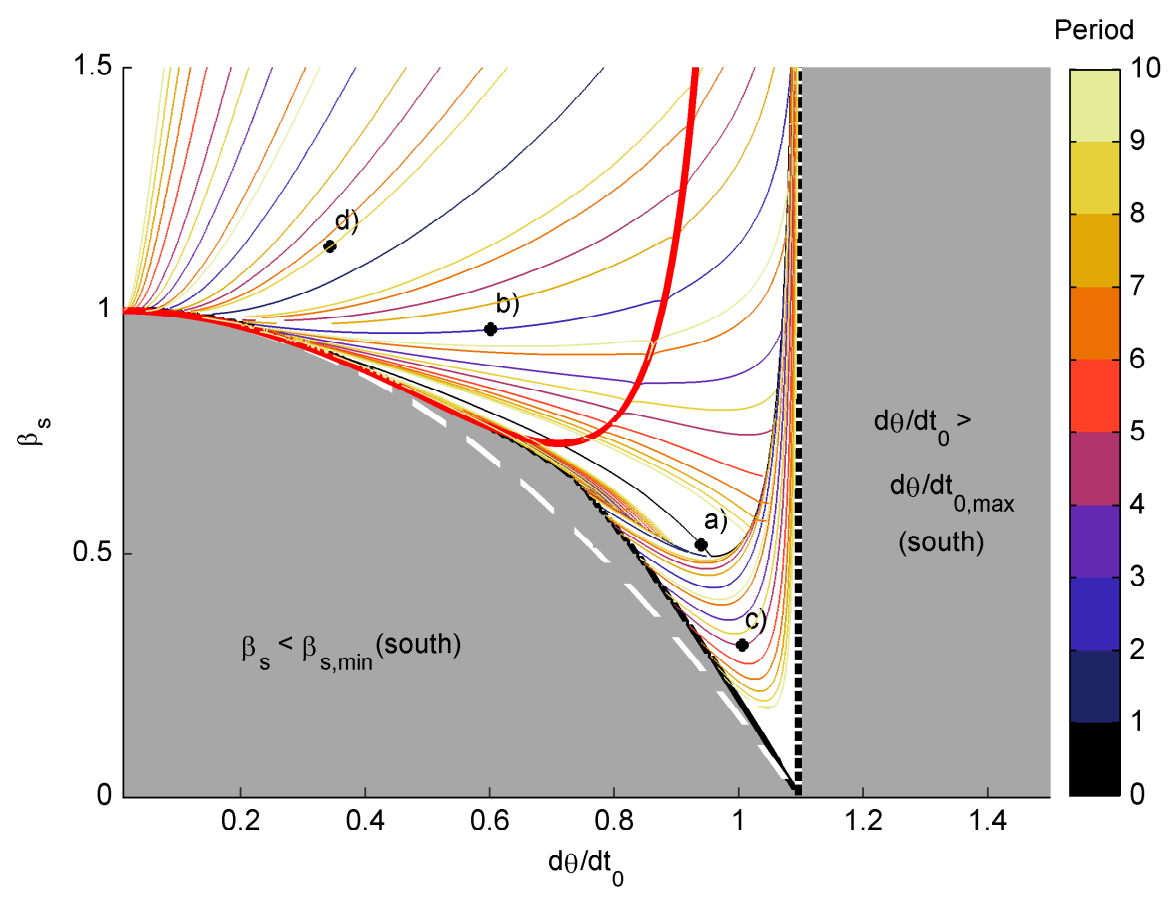

Figure 23: Feasibility region including periodic orbits for displaced, spherical NKOs with solar sail acceleration law for $\rho_{0}=0.9$ and $z_{0}=0.5$. The thick red line dividing the feasibility region represents $\beta_{s, z-s t a t i c}$. The dotted white line equals Eq. 35, the dashed white line equals Eq. 36. The black dots correspond to the orbits in Figure 24.

\subsubsection{Analysis in the $\left(\rho_{0}, z_{0}\right)$-domain}

Finally, to complete the analyses, Figure 25 shows the solar sail, periodic, spherically constrained non-Keplerian orbits in the $\left(\rho_{0}, z_{0}\right)$-domain for $d \theta / d t_{0}=1$ and $\beta_{s}=0.5$. Note that, although the initial in-plane angular velocity is equal to that of the Earth, the orbit will not be Earth-synchronous throughout its orbit since the orbit's in-plane angular velocity is not constant. Combining the results in Figure 25 with those in Figure 23 provides a complete overview of the set of solar sail, periodic, spherically constrained NKOs. 

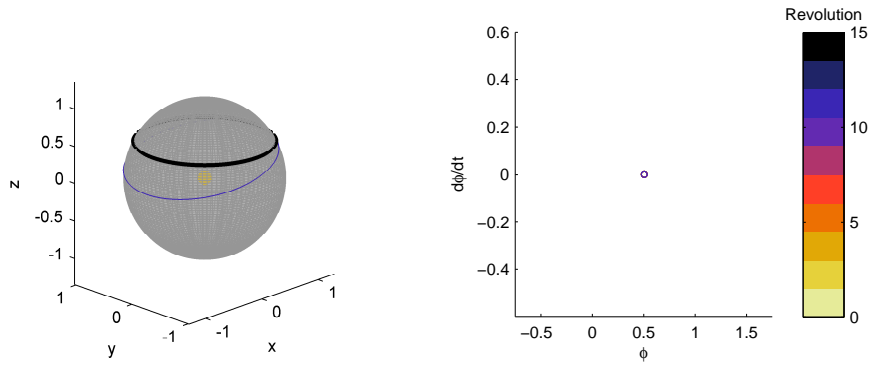

(a)
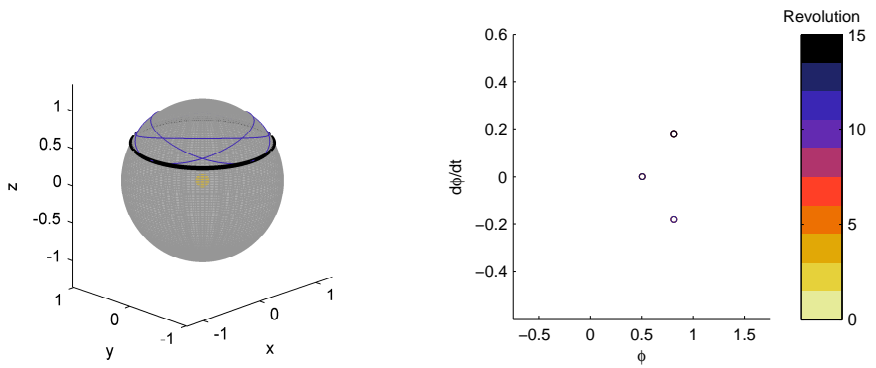

(b)
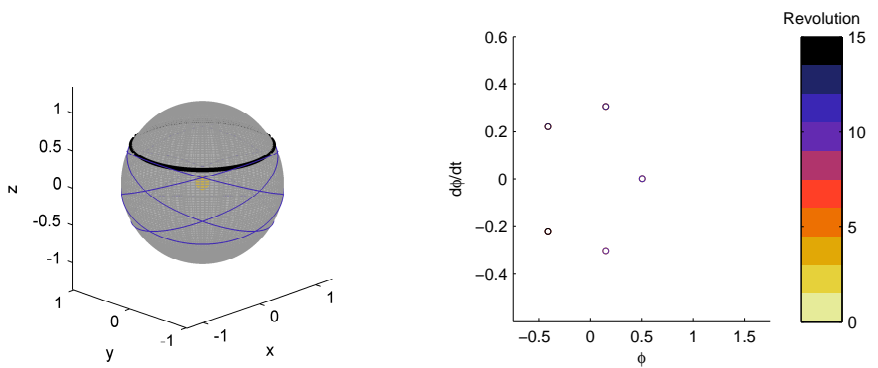

(c)
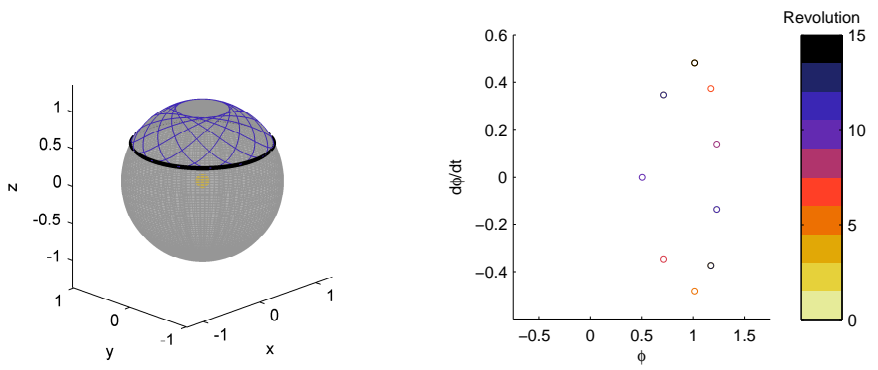

(d)

Figure 24: Orbits (see black dots in Figure 23) and Poincaré maps for displaced, spherical NKOs with solar sail acceleration law for $\rho_{0}=0.9$ and $z_{0}=0.5$. a) $d \theta / d t_{0}=0.939, \beta_{s}=0.518$. b) $d \theta / d t_{0}=0.601, \beta_{s}=0.962$. c) $d \theta / d t_{0}=1.005$, $\left.\beta_{s}=0.313 . \mathrm{d}\right) . d \theta / d t_{0}=0.343, \beta_{s}=1.132$. 
As observed for the cylindrical orbits in Section 1.2.2, also for the spherical orbits regions of clustered periodic orbits and bifurcations exist in the $\left(d \theta_{0} / d t_{0}, \beta\right)$ - and $\left(\rho_{0}, z_{0}\right)$-domains as clearly shown in the figures throughout this chapter. While Figure 15 demonstrated the existence of cylindrical periodic orbits in a clustered region, Figure 26 shows the behaviour close to a bifurcation point: a slight change in the initial out-of-plane displacement, $z_{0}$, can change the orbital period from 7 to 8 . Again, it is clear that precise insertion into the orbit as well as close navigation during the orbit is going to be essential to guarantee that the sail will stay on the targeted orbit.

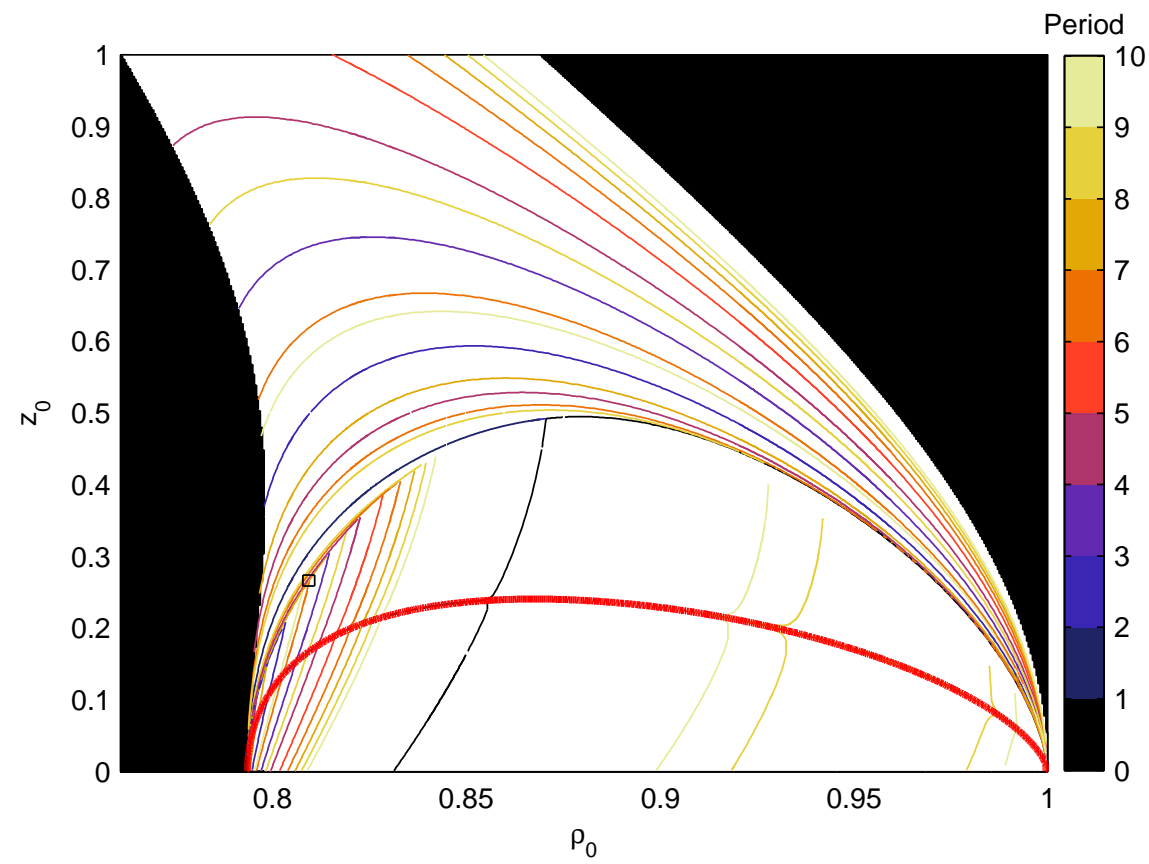

Figure 25: Feasibility region and periodic orbits in the $\left(\rho_{0}, z_{0}\right)$-domain for displaced, spherical NKOs with solar sail acceleration law for $d \theta / d t_{0}=1.0$ and $\beta_{s}=0.5$. The black box refers to the detail in Figure 26 .

\subsection{Applications}

As for the cylindrical orbits, the spherical NKOs enable the applications of the $z$-static NKOs but at a reduced required performance of the propulsion system. This is again clear from the phase spaces in Figure 21, where the dark lines on the left side of the grey transparent plane remain close to the black dots on this plane that represent the $z$-static NKOs. A good example is also given in Figure $22 \mathrm{~b}$. This orbit allows for a $27 \%$ reduction in the required scaling factor while remaining close to the $z$-static NKO. This shows again that, if a slight deviation 

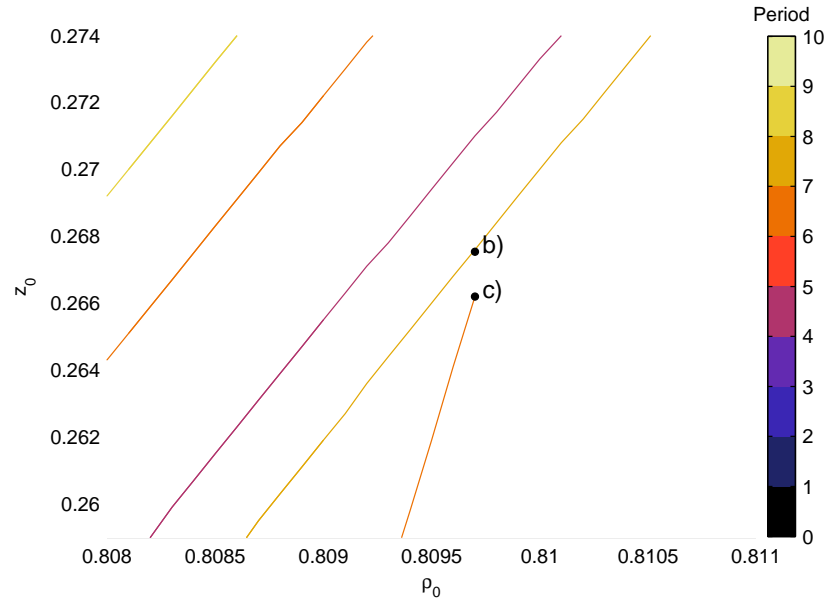

(a)
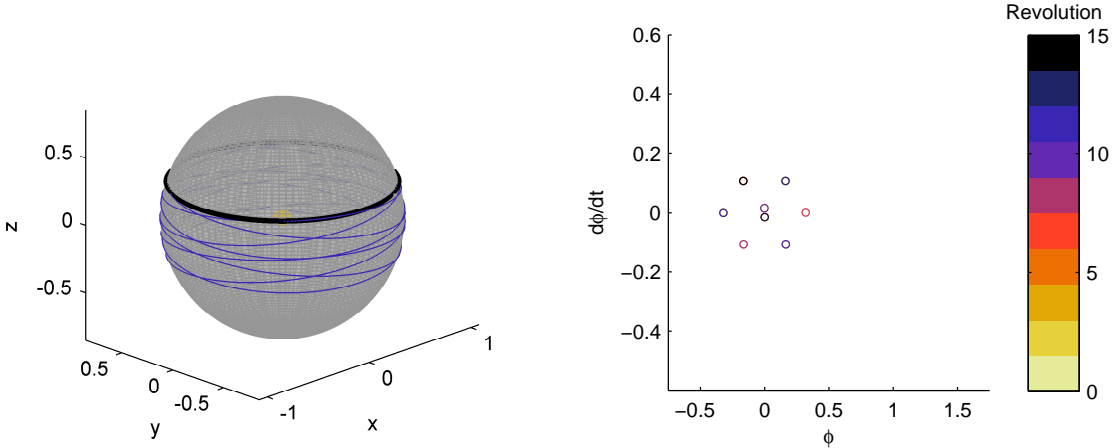

(b)
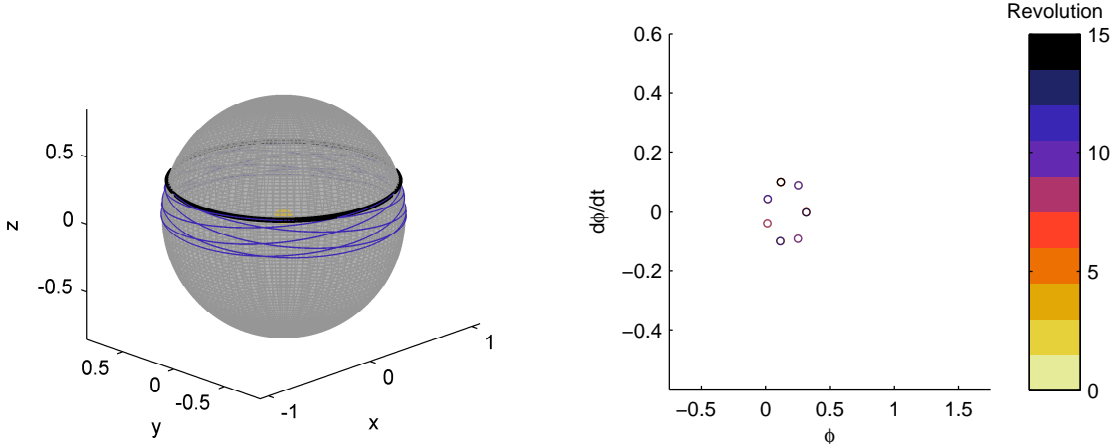

(c)

Figure 26: a) Detail of Figure 25. The black dots correspond to the orbits in subplots b-c). b) $\rho_{0}=0.8097, z_{0}=0.2675$. c) $\rho_{0}=0.8097, z_{0}=0.2662$. 
from the $z$-static NKO is allowed, the spherical NKOs could significantly reduce the required performance of the propulsion system compared to what is required for a $z$-static NKO.

More specifically, by remaining at a constant radial distance from the Sun, the spherically constrained orbits allow for some interesting solar observations. The orbits that cover the poles of the spherical surface, such as the orbits in Figure 22a and d and Figure 24b and d, would be highly suitable for solar polar observation. Since the poles of the Sun cannot be viewed from conventional orbits that traditionally lie in the ecliptic plane, these NKOs can provide new insights in the interesting physical processes that occur at the poles of the Sun. Alternatively, the orbits that cover a significant part of the surface, such as the equatorial spherical orbits and, for example, the orbit in Figure 22c, would be highly suitable for a three-dimensional mapping of the features and structures of the Sun.

\section{Conclusions}

As an extension to the well-known families of displaced non-Keplerian orbits (NKOs) that are confined to a two-dimensional plane (also referred to as " $z$ static" NKOs), this paper has introduced Sun-centred NKOs that are constrained to a three-dimensional cylindrical or spherical surface. To maintain these orbits, the use of two types of propulsion technologies have been investigated: an inverse square acceleration law (mimicking solar electric propulsion) and an ideal solar sail acceleration law. For both types of orbits and both types of propulsion, the geometrically constrained equations of motion have been derived and by setting further constraints on the in-plane angular velocity and the acceleration magnitude, sets of feasible orbits have been defined. Within these sets, true periodic orbits have been found that can serve a range of space applications: those orbits that allow a reduction in the acceleration magnitude with respect to the $z$-static NKO from which they bifurcate, but stay close to this $z$-static NKO, may provide a viable alternative to the $z$-static $\mathrm{NKO}$ as less demanding propulsion technology is required. Furthermore, spherical orbits that maintain a position high on the spherical surface are considered to be of interest for solar polar observation, while those that cover a significant part of the spherical or cylindrical surface could serve for a 3D mapping of the features and structures of the Sun.

\section{Acknowledgements}

This work was funded by the European Research Council Advanced Investigator Grant-227571: Visionary Space Systems: Orbital Dynamics at Extremes of Spacecraft Length-Scale. 


\section{References}

Baig S, McInnes C (2010) Light-levitated geostationary cylindrical orbits are feasible. Journal of Guidance, Control, and Dynamics 33(3):782-793

Biddy C, Svitek T (2012) LightSail-1 solar sail design and qualification. In: Proceedings of the 41st Aerospace Mechanisms Symposium, Pasadena, CA

Heiligers J, Ceriotti M, McInnes C, Biggs J (2011) Displaced geostationary orbit design using hybrid sail propulsion. Journal of Guidance, Control, and Dynamics 34(6):1852-1866

Johnson L, Whorton M, Heaton A, Pinson R, Laue G, Adams C (2011) NanosailD: A solar sail demonstration mission. Acta Astronautica 68:571-575

Kawaguchi J, Fujiwara A, Uesugi T (2008) Hayabusa - its technology and science accomplishment summary and Hayabusa-2. Acta Astronautica 62(1011):639647

Macdonald M, McInnes C, Alexander D, Sandman A (2006) Geosail: Exploring the magnetosphere using a low-cost solar sail. Acta Astronautica 59:757-767

McInnes C (1997) The existence and stability of families of displaced two-body orbits. Celestial Mechanics and Dynamical Astronomy 67(2):167-180

McInnes C (1998) Dynamics, stability, and control of displaced non-Keplerian orbits. Journal of Guidance, Control, and Dynamics 21(5):799-805

McInnes C (1999) Solar Sailing: Technology, Dynamics and Mission Applications. Springer-Praxis Books in Astronautical Engineering, Springer-Verlag, Berlin

McInnes C (2011) Displaced non-keplerian orbits using implisve thrust. Celestial Mechanics and Dynamical Astronomy 110(3):199-215

McInnes C, Simmons J (1992) Solar sail halo orbits. Part I - heliocentric case. Journal of Spacecraft and Rockets 29(4):466-471

Mengali G, Quarta A (2009) Non-keplerian orbits for electric sails. Celestial Mechanics and Dynamical Astronomy 105(1-3):179-195

Meserve B (1982) Fundamental Concepts of Algebra. Dover Publications, New York

Muzi D, Allasio A (2004) Goce: The first core Earth explorer of ESA's Earth observation programme. Acta Astronautica 54(3):167-175

Russell CT, Coradini A, Christensen U, De Sanctis MC, Feldman WC, Jaumann R, Keller HU, Konopliv AS, McCord TB, McFadden LA, McSween HY, Mottola S, Neukum G, Pieters CM, Prettyman TH, Raymond CA, Smith DE, Sykes MV, Williams BG, Wise J, Zuber MT (2004) Dawn: A journey in space and time. Planetary and Space Science 52(56):465-489 
Spilker T (2003) Saturn ring observer. Acta Astronautica 52:259-265

Tsuda Y, Mori O, Funase R, Sawada H, Yamamoto T, Saiki T, Endo T, Kawaguchi J (2011) Flight status of IKAROS deep space solar sail demonstrator. Acta Astronautica 69(9-10):833-840 